\title{
Deep Learning on Image Denoising: An Overview
}

\author{
Chunwei Tian ${ }^{\mathrm{a}, \mathrm{b}}$, Lunke Fei ${ }^{\mathrm{c}}$, Wenxian Zheng ${ }^{\mathrm{d}}$, Yong $\mathrm{Xu}^{\mathrm{a}, \mathrm{b}, \mathrm{e}, *}$, Wangmeng Zuo ${ }^{\mathrm{f}, \mathrm{e}}$, Chia-Wen Lin $^{\mathrm{g}}$ \\ ${ }^{a}$ Bio-Computing Research Center, Harbin Institute of Technology, Shenzhen, Shenzhen, 518055, Guangdong, China \\ ${ }^{b}$ Shenzhen Key Laboratory of Visual Object Detection and Recognition, Shenzhen, 518055, Guangdong, China \\ ${ }^{c}$ School of Computers, Guangdong University of Technology, Guangzhou, 510006, Guangdong, China \\ ${ }^{d}$ Tsinghua Shenzhen International Graduate School, Shenzhen, 518055, Guangdong, China \\ ${ }^{e}$ Peng Cheng Laboratory, Shenzhen, 518055, Guangdong, China \\ ${ }^{f}$ School of Computer Science and Technology, Harbin Institute of Technology, Harbin, 150001, Heilongjiang, China \\ ${ }^{g}$ Department of Electrical Engineering and the Institute of Communications Engineering, National Tsing Hua \\ University, Hsinchu, Taiwan
}

\begin{abstract}
Deep learning techniques have received much attention in the area of image denoising. However, there are substantial differences in the various types of deep learning methods dealing with image denoising. Specifically, discriminative learning based on deep learning can ably address the issue of Gaussian noise. Optimization models based on deep learning are effective in estimating the real noise. However, there has thus far been little related research to summarize the different deep learning techniques for image denoising. In this paper, we offer a comparative study of deep techniques in image denoising. We first classify the deep convolutional neural networks (CNNs) for additive white noisy images; the deep CNNs for real noisy images; the deep CNNs for blind denoising and the deep CNNs for hybrid noisy images, which represents the combination of noisy, blurred and low-resolution images. Then, we analyze the motivations and principles of the different types of deep learning methods. Next, we compare the state-of-the-art methods on public denoising datasets in terms of quantitative and qualitative analysis. Finally, we point out some potential challenges and directions of future research.
\end{abstract}

Keywords: Deep learning, Image denoising, Real noisy images, Blind denoising, Hybrid noisy images

\section{Introduction}

Digital image devices have been widely applied in many fields, including recognition of individuals [117, 222, 223], and remote sensing [50]. The captured image is a degraded image from the latent observation, in which the degradation processing is affected by factors such as lighting and noise corruption [265, 254]. Specifically, the noise is generated in the processes of transmission and compression from the unknown latent observation [231]. It is essential to use image denoising techniques to remove the noise and recover the latent observation from the given degraded image.

\footnotetext{
${ }^{*}$ Corresponding author

Email address: yongxu@ymail.com (Yong Xu)
} 
Image denoising techniques have attracted much attention in recent 50 years [19, 233]. At the outset, nonlinear and non-adaptive filters were used for image applications [87]. Nonlinear filters can preserve the edge information to suppress the noise, unlike linear filters [170]. Adaptive nonlinear filters depend on local signal-to-noise ratios to derive an appropriate weighting factor for removing noise from an image corrupted by the combination of additive random, signaldependent, impulse noise and additive random noise [19]. Non-adaptive filters can simultaneously use edge information and signal-to-noise ratio information to estimate the noise [82]. In time, machine learning methods, such as sparse-based methods were successfully applied in image denoising [45]. A non-locally centralized sparse representation (NCSR) method used nonlocal selfsimilarity to optimize the sparse method, and obtained high performance for image denoising [49]. To reduce computational costs, a dictionary learning method was used to quickly filter the noise [54]. To recover the detailed information of the latent clean image, priori knowledge (i.e., total variation regularization) can smooth the noisy image in order to deal with the corrupted image [163, 178]. More competitive methods for image denoising can be found in [150, 275, 258], including the Markov random field (MRF) [184], the weighted nuclear norm minimization (WNNM) [69], learned simultaneous sparse coding (LSSC) [150], cascade of shrinkage fields (CSF) [184], trainable nonlinear reaction diffusion (TNRD) [35] and gradient histogram estimation and preservation (GHEP) [275].

Although most of the above methods have achieved reasonably good performance in image denoising, they suffered from several drawbacks [146], including the need for optimization methods for the test phase, manual setting parameters, and a certain model for single denoising tasks. Recently, as architectures became more flexible, deep learning techniques gained the ability to overcome these drawbacks [146].

The original deep learning technologies were first used in image processing in the 1980s [60] and were first used in image denoising by Zhou et al. [38, 273]. That is, the proposed denoising work first used a neural network with both the known shift-invariant blur function and additive noise to recover the latent clean image. After that, the neural network used weighting factors to remove complex noise [38]. To reduce the high computational costs, a feedforward network was proposed to make a tradeoff between denoising efficiency and performance [198]. The feedforward network can smooth the given corrupted image by Kuwahara filters, which were similar to convolutions. In addition, this research proved that the mean squared error (MSE) acted as a loss function and was not unique to neural networks [48, 68]. Subsequently, more optimization algorithms were used to accelerate the convergence of the trained network and to promote the denoising performance [16, 47, 61]. The combination of maximum entropy and prima-dual Lagrangian multipliers to enhance the expressive ability of neural networks proved to be a good tool for image denoising [15]. To further make a tradeoff between fast execution and denoising performance, greedy algorithms and asynchronous algorithms were applied in neural networks [164]. Alternatively, designing a novel network architecture proved to be very competitive in eliminating the noise, through either increasing the depth or changing activation function [190]. Cellular neural networks (CENNs) mainly used nodes with templates to obtain the averaging function and effectively suppress the noise [190, 161]. Although this proposed method can obtain good denoising results, it requires the parameters of the templates to be set manually. To resolve this problem, the gradient descent was developed [252, 114]. To a certain degree, these deep techniques can im- 
prove denoising performance. However, these networks did not easily allow the addition of new plug-in units, which limited their applications in the real world [59].

Based on the reasons above, convolutional neural networks (CNNs) were proposed [141, 180]. The CNN as well as the LeNet had real-world application in handwritten digit recognition [113]. However, due to the following drawbacks, they were not widely applied in computer systems [109]. First, deep CNNs can generate vanishing gradients. Second, activation functions such as sigmoid [154] and tanh [92] resulted in high computational cost. Third, the hardware platform did not support the complex network. However, that changed in 2012 with AlexNet in that year's ImageNet Large-Scale Visual Recognition Challenge (ILSVRC) [109]. After that, deep network architectures (e.g., VGG [189] and GoogLeNet [196]) were widely applied in the fields of image [225, 217, 119], video [138, 248], nature language processing [52] and speech processing [267], especially low-level computer vision [169, 204].

Deep networks were first applied in image denoising in 2015 [129, 234]. The proposed network need not manually set parameters for removing the noise. After then, deep networks were widely applied in speech [268], video [247] and image restoration [209, 176]. Mao et al. [152] used multiple convolutions and deconvolutions to suppress the noise and recover the high-resolution image. For addressing multiple low-level tasks via a model, a denoising CNN (DnCNN) [258] consisting of convolutions, batch normalization (BN) [89], rectified linear unit (ReLU) [159] and residual learning (RL) [75] was proposed to deal with image denoising, super-resolution, and JPEG image deblocking. Taking into account the tradeoff between denoising performance and speed, a color non-local network (CNLNet) [116] combined non-local self-similarity (NLSS) and CNN to efficiently remove color-image noise.

In terms of blind denoising, a fast and flexible denoising CNN (FFDNet) [260] presented different noise levels and the noisy image patch as the input of a denoising network to improve denoising speed and process blind denoising. For handling unpaired noisy images, a generative adversarial network (GAN) CNN blind denoiser (GCBD) [31] resolved this problem by first generating the ground truth, then inputting the obtained ground truth into the GAN to train the denoiser. Alternatively, a convolutional blind denoising network (CBDNet) [71] removed the noise from the given real noisy image by two sub-networks, one in charge of estimating the noise of the real noisy image, and the other for obtaining the latent clean image. For more complex corrupted images, a deep plug-and-play super-resolution (DPSR) method [262] was developed to estimate blur kernel and noise, and recover a high-resolution image. Although other important research has been conducted in the field of image denoising in recent years, there have been only a few reviews to summarize the deep learning techniques in image denoising [205]. Although Ref. [205] referred to a good deal work, it lacked more detailed classification information about deep learning for image denoising. For example, related work pretaining to unpaired real noisy images was not covered. To this end, we aim to provide an overview of deep learning for image denoising, in terms of both applications and analysis. Finally, we discuss the state-of-the-art methods for image denoising, including how they can be further expanded to respond to the challenges of the future, as well as potential research directions. An outline of this survey is shown in Fig. 1.

This overview covers more than 200 papers about deep learning for image denoising in recent years. The main contributions in this paper can be summarized as follows.

1. The overview illustrates the effects of deep learning methods on the field of image denoising. 


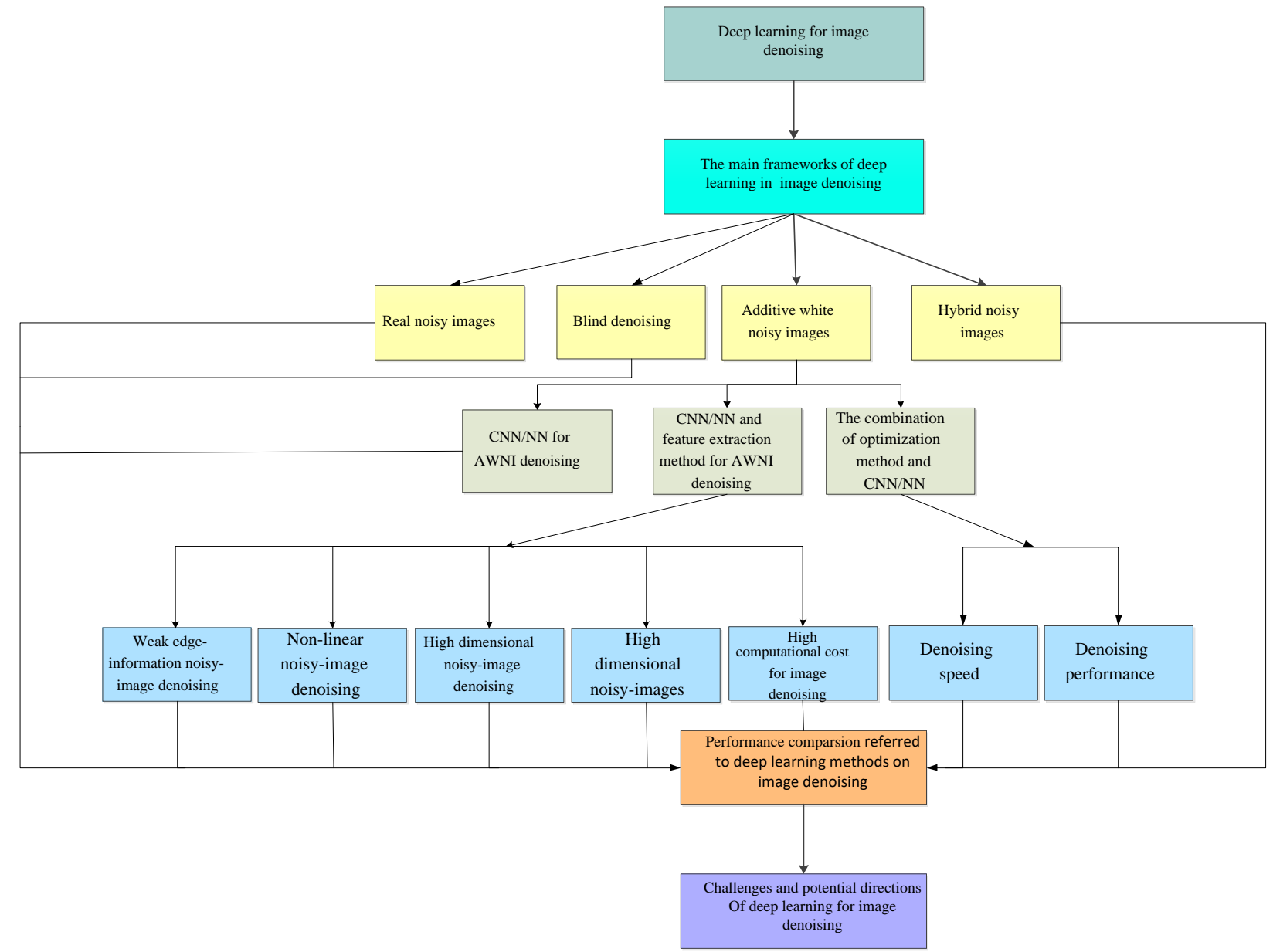

Figure 1: Outline of the survey. It consists of four parts, including basic frameworks, categories, performance comparison, challenges and potential directions. Specifically, categories comprise additive white noisy images, real noisy images, blind denoising and hybrid noisy images.

2. The overview summarizes the solutions of deep learning techniques for different types of noise (i.e., additive white noise, blind noise, real noise and hybrid noise) and analyzes the motivations and principles of these methods in image denoising, where blind noise denotes noise of unknown types. Finally, we evaluate the denoising performance of these methods in terms of quantitative and qualitative analysis.

3. The overview points out some potential challenges and directions for deep learning in the use of image denoising.

The rest of this overview is organized as followed.

Section 2 discusses the popular deep learning frameworks for image applications. Section 3 presents the main categories of deep learning in image denoising, as well as a comparison and analysis of these methods. Section 4 offers a performance comparison of these denoising methods. Section 5 discusses the remaining challenges and potential research directions. Section 6 offers the authors' conclusions. 


\section{Fundamental frameworks of deep learning methods for image denoising}

This section offers a discussion of deep learning, including the ideas behind it, the main network frameworks (techniques), and the hardware and software, which is the basis for the deep learning techniques for image denoising covered in this survey.

\subsection{Machine learning methods for image denoising}

Machine learning methods consist of supervised, semi-supervised and unsupervised learning methods. Supervised learning methods [133, 228, 120] use the given label to put the obtained features closer to the target for learning parameters and training the denoising model. For example, take a given denoising model $y=x+\mu$, where $x, y$ and $\mu$ represent the given clean image, noisy image and additive Gaussian noise (AWGN) of standard deviation $\sigma$, respectively. From the equation above and Bayesian knowledge, it can be seen that the learning of parameters of the denoising model relies on pair $\left\{x_{k}, y_{k}\right\}_{k=1}^{N}$, where $x_{k}$ and $y_{k}$ denote the $k t h$ clean image and noisy image, respectively. Also, $N$ is the number of noisy images. This processing can be expressed as $x_{k}=f\left(y_{k}, \theta, m\right)$, where $\theta$ is the parameters and $m$ denotes the given noise level.

Unsupervised learning methods [115] use given training samples to find patterns rather than label matching and finish specific tasks, such as unpairing real low-resolution images [250]. The recently proposed Cycle-in-Cycle GAN (CinCGAN) recovered a high-resolution image by first estimating the high-resolution image as a label, then exploiting the obtained label and loss function to train the super-resolution model.

Semi-supervised learning methods [40] apply a model from a given data distribution to build a learner for labeling unlabeled samples. This mechanism is favored by small sample tasks, such as medical diagnosis. A semi-supervised learned sinogram restoration network (SLSR-Net) can learn feature distribution from paired sinograms via a supervised network, and then, convert the obtained feature distribution to a high-fidelity sinogram from unlabeled low-dose sinograms via an unsupervised network [157].

\subsection{Neural networks for image denoising}

Neural networks are the basis of machine learning methods, which in turn are the basis of deep learning techniques [183]. Most neural networks consist of neurons, input $X$, activation function $f$, weights $W=\left[W^{0}, W^{1}, \ldots, W^{n-1}\right]$ and biases $b=\left[b^{0}, b^{1}, \ldots, b^{n}\right]$. The activation functions such as Sigmoid [154, 104] and tanh [92, 55] can convert the linear input into non-linearity through $W$ and $b$ as follows.

$$
f(X ; W ; b)=f\left(W^{T} X+b\right) .
$$

Note that if the neural network has multiple layers, it is regarded as multilayer perceptron (MLP) [25]. In addition, the middle layers are treated as hidden layers beside the input and output layers. This process can be expressed as

$$
f(X ; W ; b)=f\left(W^{n} f\left(W^{n-1} \ldots f\left(W^{0} X+b^{0}\right) \ldots b^{n-1}\right)+b^{n}\right),
$$

where $n$ is the final layer of the neural network. To help readers understand the principle of the neural network, a visual example is provided in Fig. 2. 


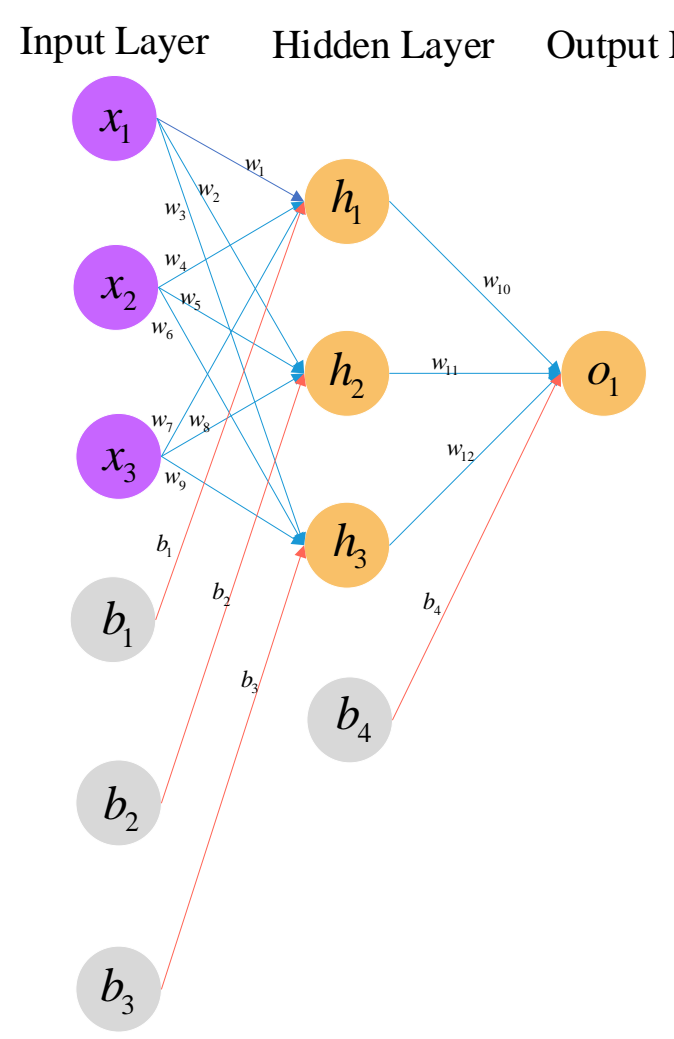

Figure 2: Two-layer neural network.

The two-layer fully connected neural network includes two layers: a hidden layer and output layer (the input layer is not generally regarded as a layer of a neural network). There are parameters to be defined: $x_{1}, x_{2}, x_{3}$ and $o_{1}$ represent the inputs and output of this neural network, respectively. $w_{1}, w_{2}, \ldots, w_{11}, w_{12}$ and $b_{1}, b_{2}, b_{3}, b_{4}$ are the weights and biases, respectively. For example, the output of one neuron $h_{1}$ via Eqs. (3) and (4) is obtained as follows:

$$
\begin{gathered}
f\left(z_{h 1}\right)=f\left(w_{1} x_{1}+w_{4} x_{2}+w_{7} x_{3}+b_{1}\right) . \\
o\left(h_{1}\right)=f\left(z_{h 1}\right) .
\end{gathered}
$$

First, the output of the network $o_{1}$ is obtained. Then, the network uses back propagation (BP) [81] and loss function to learn parameters. That is, when the loss value is within specified limitation, the trained model is considered as well-trained. It should be noted that if the number of layers of a neural network is more than three, it is also referred to as a deep neural network. Stacked auto-encoders (SARs) [80] and deep belief networks (DBNs) [17, 79] are typical deep neural networks. They used stacked layers in an unsupervised manner to train the models and obtain good performance. However, these networks are not simple to implement and require a good deal of manual settings to achieve an optimal model. Due to this, end-to-end connected networks, especially CNNs, were proposed [241]. CNNs have wide applications in the field of image processing, especially image denoising. 


\subsection{CNNs for image denoising}

Due to their plug-and-play network architectures, CNNs have achieved great success in image processing [257, 145, 121]. As a pioneer in CNN technology, LeNet [113] used convolutional kernels of different sizes to extract features and obtain good performance in image classification. However, due to the Sigmoid activation function, LeNet had a slow convergence speed, which was a shortcoming in real-world applications.

After LeNet, the proposed AlexNet [109] was a milestone for deep learning. Its success was due to several reasons. First, the graphics processing unit (GPU) [154] provided strong computational ability. Second, random clipping (i.e., dropout) solved the overfitting problem. Third, ReLU [159] improved the speed of stochastic gradient descent (SGD) rather than Sigmoid [22]. Fourth, the data augmentation method further addressed the overfitting problem. Although AlexNet achieved good performance, it required substantial memory usage due to its large convolutional kernels. That limited its real-world applications, such as in smart cameras. After that, during the period of 2014 to 2016, deeper network architectures with small filters were preferred to improve the performance and reduce computational costs. Specifically, VGG [189] stacked more convolutions with small kernel sizes to win the ImageNet LSVR Challenge in 2014. Fig. 3 depicts the network architecture.

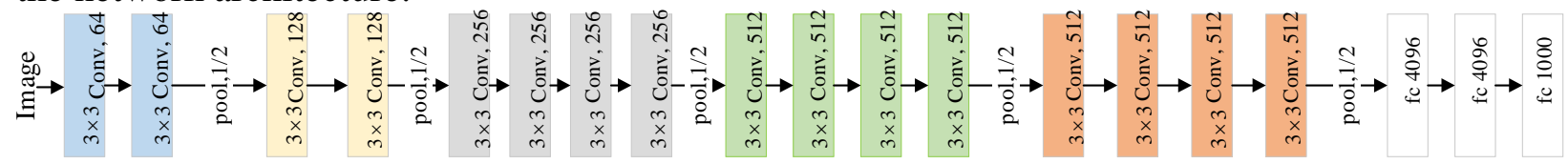

Figure 3: Network architecture of VGG.

With the success of deeper networks, the research turned to increasing their width. GoogLeNet [196] increased the width to improve the performance for image applications. Moreover, GoogLeNet transformed a large convolutional kernel into two smaller convolution kernels in order to reduce the number of parameters and computational cost. GoogLeNet also used the inception module [132] as well as Inception 1. Its visual network figure is shown in Fig. 4.

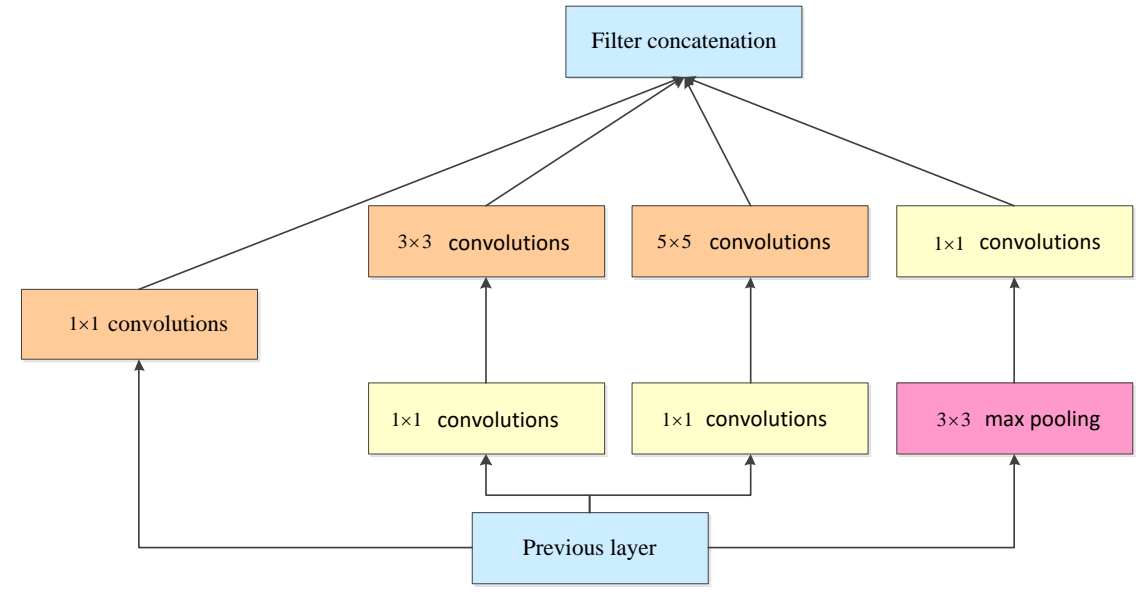

Figure 4: Network architecture of GoogLeNet (Inception 1).

Although VGG and GoogLeNet methods are effective for image applications, they have two drawbacks: if the network is very deep, this may result in vanishing or exploding gradients; and if 
the network is very wide, it may be subject to the phenomenon of overfitting. To overcome these problems, ResNet [75] was proposed in 2016. Each block was given by adding residual learning operation in ResNet to improve the performance of image recognition, which leads to ResNet winning the mageNet LSVR in 2015. Fig. 5 depicts the concept of residual learning.

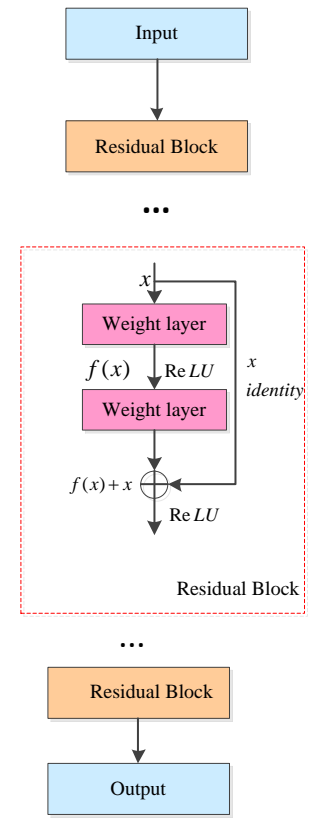

Figure 5: Network architecture of ResNet.

Since 2014, deep networks have been widely used in real-world image applications, such as facial recognition [86] and medical diagnosis [124]. However, in many applications, captured images, such as real noisy images, are not sufficient, and deep CNNs tend to perform poorly in image applications. For this reason, GANs [173] were developed. GANs consisted of two networks: generative and discriminative networks. The generative network (also referred to as the generator) is used to generate samples, according to input samples. The discriminative network (also called the discriminator) is used to judge the truth of both input samples and generated samples. The two networks are adversarial. Note that if the discriminator can accurately distinguish real samples and generate samples from generator, the trained model is regarded as finished. The network architecture of the GAN can be seen in Fig. 6. Due to its ability to construct supplemental training samples, the GAN is very effective for small sample tasks, such as facial recognition [211] and complex noisy image denoising [31]. These mentioned CNNs are basic networks for image denoising.

\subsection{Hardware and software used in deep learning}

One reason for the success of deep learning is the GPU. The GPU uses the CUDA [162], OpenCL [193] and cuDNN [37] platforms to strengthen its parallel computing ability, which exceeds the speed of the CPU by 10 to 30 times. The GPU consists of an NVIDIA consumer line of graphics cards (i.e., GTX 680, GTX 980, GTX 1070, GTX 1070Ti, GTX1080, GTX 1080Ti, RTX 2070, RTX 2080, RTX 2080Ti, Tesla K40c, Tesla K80, Quadro M6000, Quadro GP100, Quadro P6000 and Tesla V100) and AMD (i.e., Radeon Vega 64 and FE) [110]. 


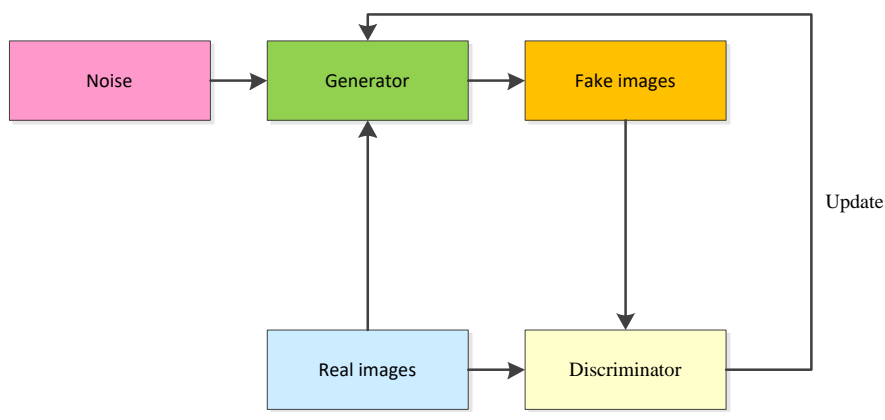

Figure 6: Network architecture of GAN.

Deep learning software can provide interfaces to call the GPU. Popular software packages include:

(1) Caffe [96] based on $\mathrm{C}++$, provides $\mathrm{C}++$, Python and Matlab interfaces, which can also run on both the CPU and GPU. It is widely used for object detection tasks. However, it requires developers to master $\mathrm{C}++$.

(2) Theano [18] is a compiler of math expressions for dealing with large-scale neural networks. Theano provides a Python interface and is used in image super-resolution, denoising and classification.

(3) Matconvnet [214] offers the Matlab interface. It is utilized in image classification, denoising and super-resolution, and video tracking. However, it requires Matlab mastery.

(4) TensorFlow [1] is a relatively high-order machine learning library. It is faster than Theano for compilation. TensorFlow offers $\mathrm{C}++$ and Python interfaces and is used in object detection, image classification, denoising and super-resolution.

(5) Keras [41] based on TensorFlow and Theano is implemented in Python and offers a Python interface. It can be used in image classification, object detection, image resolution, image denoising and action recognition.

(6) PyTorch [168] is implemented in Python and offers a Python interface. It is employed in image classification, object detection, image segmentation, action recognition, image superresolution, image denoising and video tracking.

\section{Deep learning techniques in image denoising}

\subsection{Deep learning techniques for additive white noisy-image denoising}

Due to the insufficiency of real noisy images, additive white noisy images (AWNIs) are widely used to train the denoising model [102]. AWNIs include Gaussian, Poisson, Salt, Pepper and multiplicative noisy images [56]. There are several deep learning techniques for AWNI denoising, including CNN/NN; the combination of CNN/NN and common feature extraction methods; and the combination of the optimization method and CNN/NN.

\subsubsection{CNN/NN for AWNI denoising}

Automatic feature extraction methods can play a major role in reducing the computational costs for image applications [240, 179, 143]. For this reason, CNNs have been developed for image denoising [155, 134]. Zhang et al. [258] proposed a model as well as a DnCNN to deal with 
Table 1: CNN/NN for AWNI denoising.

\begin{tabular}{|c|c|c|c|}
\hline References & Methods & Applications & Key words (remarks) \\
\hline $\begin{array}{lll}\text { Zhang et al. (2017) 258 } \\
\end{array}$ & CNN & Gaussian image denoising, super-resolution and JPEG deblocking & CNN with residual learning, and BN for image denoising \\
\hline Wang et al. (2017) 219] & CNN & Gaussian image denoising & CNN with dilated convolutions, and BN for image denoising \\
\hline Bae et al. (2017) 13 & CNN & Gaussian image denoisng, super-resolution & CNN with wavelet domain, and residual learning $(\mathrm{RL})$ for image restoration \\
\hline Jin et al. (2017) 101 & CNN & Medical (X-ray) image restoration & Improved Unet from iterative shrinkage idea for medical image restoration \\
\hline Tai et al. (2017) 197] & CNN & Gaussian image denoisng, super-resolution and JPEG deblocking & CNN with recursive unit, gate unit for image restoration \\
\hline Anwar et al. (2017) [12] & CNN & Gaussian image denoisng & CNN with fully connected layer, RL and dilated convolutions for image denoising \\
\hline McCann et al. (2017)] [102] & CNN & Inverse problems (i.e., denoising, deconvolution, super-resolution) & CNN for inverse problems \\
\hline Ye et al. (2018) [242] & CNN & Inverse problems(i.e., Gaussian image denoising, super-resoluion) & Signal processing ideas guide $\mathrm{CNN}$ for inverse problems \\
\hline Yuan et al. (2018) [249] & CNN & Hyper-spectral image denoising & CNN with multiscale, multilevel features techniques for hyper-spectral image denoising \\
\hline Jiang et al. (2018) [98] & CNN & Gaussian image denoising & Multi-channel CNN for image denoising \\
\hline Chang et al. (2018) 28] & CNN & Hyper-spectral image (HSI) denoising, HIS restoration & CNN consolidated spectral and spatial coins for hyper-spectral image denoising \\
\hline Jeon et al. (2018) [93] & CNN & Speckle noise reduction from digital holographic images & Speckle noise reduction of digital holographic image from Multi-scale CNN \\
\hline Gholizadeh-Ansari et al. (2018) 63] & CNN & Low-dose CT image denoising, X-ray image denosing & CNN with dilated convolutions for low-dose CT image denoising \\
\hline Uchida et al. (2018) 213] & CNN & Non-blind image denoising & CNN with residual learning for non-blind image denoising \\
\hline Xiao et al. (2018) [227] & CNN & Stripe noise reduction of infrared cloud images & CNN with skip connection for infrared-cloud-image denoising \\
\hline Chen et al. (2018) 34] & CNN & Gaussian image denoisng, blind denoising & CNN based on RL and perceptual loss for edge enhancement \\
\hline Yu et al. (2018) 246 & CNN & Seismic, random, linear and multiple noise reduction of images & A survey on deep learning for three applications \\
\hline Yu et al. (2018) 245 & CNN & Optical coherence tomography (OCT) image denoising & GAN with dense skip connection for optical coherence tomography image denoising \\
\hline Li et al. (2018) 118 & CNN & Ground-roll noise reduction & An overview of deep learning techniques on ground-roll noise \\
\hline Abbasi et al. (2018) [2] & CNN & OCT image denoising & Fully CNN with multiple inputs, and RL for OCT image denoising \\
\hline Zarshenas et al. (2018) [253] & CNN & Gaussian noisy image denoising & Deep CNN with internal and external residual learning for image denoising \\
\hline Chen et al. (2018) 29] & CNN & Gaussian noisy image denoising & CNN with recursive operations for image denoising \\
\hline Panda et al. (2018) [165 & CNN & Gaussian noisy image denoising & CNN with exponential linear units, and dilated convolutions for image denoising \\
\hline Sheremet et al. (2018) 186$]$ & CNN & Image denoising from info-communication systems & CNN on image denoising from info-communication systems \\
\hline Chen et al. (2018) [30] & CNN & Aerial-image denoising & CNN with multi-scale technique, and RL for aerial-image denoising \\
\hline Pardasani et al. (2018) 166$]$ & CNN & Gaussian, poisson or any additive-white noise reduction & CNN with BN for image denoising \\
\hline Couturier et al. (2018) 42] & NN & Gaussian and multiplicative speckle noise reduction & Encoder-decoder network with multiple skip connections for image denoising \\
\hline Park et al. (2018) [167] & CNN & Gaussian noisy image denoising & CNN with dilated convolutions for image denoising \\
\hline Priyanka et al. (2019) [172] & CNN & Gaussian noisy image denoisng & CNN with symmetric network architecture for image denoising \\
\hline Lian et al. (2019) [194] & CNN & Poisson-noise-image denoising & CNN with multi scale, and multiple skip connections for Poisson image denoising \\
\hline Tripathi et al. (2018) 212] & CNN & Gaussian noisy image denoising & GAN for image denoising \\
\hline Zheng et al. (2019) 271] & CNN & Gaussian noisy image denoising & CNN for image denoising \\
\hline Tian et al. (2019) [204] & CNN & Gaussian noisy image denoising & CNN for image denoising \\
\hline Remez et al. (2018) 175 & CNN & Gaussian and Poisson image denoising & CNN for image denoising \\
\hline Tian et al. (2020) 207] & CNN & Gaussian image denoising and real noisy image denoising & CNN with BRN, RL and dilated convolutions for image denosing \\
\hline Tian et al. (2020) 206 & CNN & Gaussian image denoising, blind denoising and real noisy image denoising & CNN with attention mechanism and sparse method for image denoising \\
\hline Tian et al. (2020) [208] & CNN & Gaussian image denoising, blind denoising and real noisy image denoising & Two CNNs with sparse method for image denoising \\
\hline
\end{tabular}

multiple low-level vision tasks, i.e., image denoising, super-resolution and deblocking through CNN, batch normalization [89] and residual learning techniques [75]. Wang et al. [219], Bae et al. [13] and Jifara et al. [101] also presented a residual learning into deeper CNN for image denoising. However, the deeper CNN technique relied on a deeper layer rather than a shallow layer, which resulted in a long-term dependency problem. Several signal-base methods were proposed to resolve this problem. Tai et al. [197] exploited recursive and gate units to adaptively mine more accurate features and recover clean images. Inspired by a low-rank Hankel matrix in low-level vision, Ye et al. [242] provided convolution frames to explain the connection between signal processing and deep learning by convolving local and nonlocal bases. For solving insufficient noisy images (i.e., hyperspectral and medical images), several recent works have attempted to extract more useful information through the use of improved CNNs [28, 77, 245, 139]. For example, Yuan et al. [249] combined a deep CNN, residual learning and multiscale knowledge to remove the noise from hyperspectral-noisy images. However, these proposed CNNs led to the likelihood of increased computational costs and memory consumption, which was not conducive for real-world applications. To address this phenomenon, Gholizadeh et al. [63] utilized dilated convolutions [62] to enlarge the receptive field and reduce the depth of network without incurring extra costs for CT image denoising. Lian et al. [194] proposed a residual network via multi-scale cross-path concatenation to suppress the noise. Most of the above methods relied on improved CNNs to deal with the noise. Therefore, designing network architectures is important for image denoising [167, 118]. 
Changing network architectures involves the following methods [246, 149, 177]: fusing features from multiple inputs of a $\mathrm{CNN}$; changing the loss function; increasing depth or width of the $\mathrm{CNN}$; adding some auxiliary plug-ins into CNNs; and introducing skip connections or cascade operations into CNNs. Specifically, the first method includes three types: different parts of one sample as multiple inputs from different networks [2]; different perspectives for the one sample as input, such as multiple scales [93, 30]; and different channels of a $\mathrm{CNN}$ as input [98]. The second method involves the design of different loss functions according to the characteristics of nature images to extract more robust features [10]. For example, Chen et al. [34] jointed Euclidean and perceptual loss functions to mine more edge information for image denoising. The third method enlarged the receptive field size to improve denoising performance via increasing the depth or width of the network [213, 253, 186]. The fourth method applied plug-ins, such as activation function, dilated convolution, fully connected layer and pooling operations, to enhance the expressive ability of the CNN [165, 172, 166]. The fifth method utilized skip connections [227, 29, 42, 12] or cascade operations [194, 36] to provide complementary information for the deep layer in a CNN. Table 1 provides an overview of CNNs for AWNI denoising.

\subsubsection{CNN/NN and common feature extraction methods for AWNI denoising}

Feature extraction is used to represent the entire image in image processing, and it is important for machine learning [130, 144, 238]. However, because deep learning techniques are black box techniques, they do not allow the choice of choose features, and therefore cannot guarantee that the obtained features are the most robust [187, 221]. Motivated by problem, researches embedded common feature extraction methods into CNNs for the purpose of image denoising. They did this for five reasons: weak edge-information noisy images, non-linear noisy images, high dimensional noisy images and non-salient noisy images, and high computational costs.

For weak edge-information noisy images, CNN with transformation domain methods were proposed by Guan et al. [70], Li et al. [122], Liu et al. [137], Latif et al. [111] and Yang et al. [237]. However, they were not effective in removing the noise. Specifically, in [137], the proposed solution used the wavelet method and U-net to eliminate the gridding effect of dilated convolutions on enlarging the receptive field for image restoration.

For non-linear noisy images, CNNs with kernel methods proved useful [14, 235]. These methods mostly consisted of three steps [158]. The first step used CNN to extract features. The second step utilized the kernel method to convert obtained non-linear features into linearity. The third step exploited the residual learning to construct the latent clean image.

For high dimensional noisy images, the combination of CNN and the dimensional reduction method was proposed [229, 72]. For example, Khaw et al. [106] used a CNN with principal component analysis (PCA) for image denoising. This consisted of three steps. The first step used convolution operations to extract features. The second step utilized the PCA to reduce the dimension of the obtained features. The third step employed convolutions to deal with the obtained features from the PCA and to reconstruct a clean image.

For non-salient noisy images, signal processing can guide the $\mathrm{CNN}$ in extracting salient features [94, 103, 174, 2]. Specifically, skip connection is a typical operation of signal processing [103]. 
Table 2: CNN/NN and common feature extraction methods for AWNI denoising.

\begin{tabular}{|c|c|c|c|}
\hline References & Methods & Applications & Key words (remarks) \\
\hline Bako et al. (2017) [194] & CNN & Monte Carlo-rendered images denoising & CNN with kernel method for estimating noise piexls \\
\hline Ahn et al. (2017) 7$]$ & CNN & Gaussian image denoising & CNN with NSS for image denoising \\
\hline Khaw et al. (2017) 106 & CNN & Impulse noise reduction & CNN with PCA for image denoising \\
\hline Vogel et al. (2017) 215 & CNN & Gaussian image denoising & U-net with multi scales technique for image denoising \\
\hline Mildenhall et al. (2018) [158] & NN & Low-light synthetic noisy image denoising, real noise & Encoder-decoder with multi scales, and kernel method for image denoising \\
\hline Liu et al. (2018) 137 & CNN & Gaussian image denoisng, super-resolution and JPEG deblocking & U-net with wavelet for image restoration \\
\hline Yang et al. (2018) 237] & CNN & Gaussian image denoisng & CNN with BM3D for image denoising \\
\hline Guo et al. (2018) 72] & CNN & Image blurring and denoising & CNN with RL, and sparse method for image denoising \\
\hline Jia et al. (2018) 94 & CNN & Gaussian image denoisng & CNN with multi scales, and dense RL operations for image denoising \\
\hline Ran et al. (2018) [174] & CNN & OCT image denoising, OCT image super-resolution & CNN with multi views for image restoration \\
\hline Li et al. (2018) [140] & CNN & Medical image denoising, stomach pathological image denoising & CNN consolidated wavelet for medical image denoising \\
\hline Ahn et al. (2018) 8 & CNN & Gaussian image denoisng & CNN with NSS for image denoising \\
\hline Xie et al. (2018) 229] & CNN & Hyper-spectral image denoising & CNN with RL, and PCA for low-dose CT image denoising \\
\hline Kadimesetty et al. (2019) [103] & CNN & Low-Dose computed tomography (CT) image denoising & CNN with RL, batch normalization (BN) for medical image denoising \\
\hline Guan et al. (2019) [70] & CNN & Stripe noise reduction & CNN with wavelet-image denoising \\
\hline Abbasi et al. (2019) [2] & NN & 3D magnetic resonance image denoising, medical image denoising & GAN based on encoder-decoder and RL for medical denoising \\
\hline Xu et al. (2019) 235 & CNN & Synthetic and real noisy and video denoising & CNN based on deformable kernel for image and video denoising \\
\hline
\end{tabular}

For tasks involving high computational costs, a CNN with relations nature of pixels from an image was very effective in decreasing complexity [2, 8, 7]. For example, Ahn et al. [7] used a CNN with non-local self-similarity (NSS) to filter the noise, where similar characteristics of the given noisy image can accelerate the speed of extraction feature and reduce computational costs.

More detailed information on these methods mentioned can be found in Table 2.

\subsubsection{Combination of optimization method and CNN/NN for AWNI denoising}

Machine learning uses optimization techniques [85, 126] and discriminative learning methods [125, 135] to deal with image applications. Although optimization methods have good performance on different low-level vision tasks, these methods need manual setting parameters, which are time-consuming [209, 210]. The discriminative learning methods are fast in image restoration. However, they are not flexible for low-level vision tasks. To achieve a tradeoff between efficiency and flexibility, a discriminative learning optimization-based method [156, 20] was presented for image applications, such as image denoising. CNNs with prior knowledge via regular term of loss function is a common method in image denoising [83], which can be divided two categories: improvement of denoising speed and improvement of denoising performance.

For improving denoising speed, an optimization method using a CNN was an effective tool for rapidly finding the optimal solution in image denoising [39, 58]. For example, a GAN with the maximum a posteriori (MAP) method was used to estimate the noise and deal with other tasks, such as image inpainting and super-resolution [243]. An experience-based greed algorithm and transfer learning strategies with a CNN can accelerate a genetic algorithm to obtain a clean image [136]. Noisy image and noise level mapping were inputs of the $\mathrm{CNN}$, which had faster execution in predicting the noise [202]. 
Table 3: The combination of the optimization method and CNN/NN for AWNI denoising.

\begin{tabular}{|c|c|c|c|}
\hline References & Methods & Applications & Key words (remarks) \\
\hline Hong et al. (2018) [83] & CNN & Gaussian image denoising & Auto-Encoder with BN, and ReLU for image denoising \\
\hline Cho et al. (2018) 39] & CNN & Gaussian image denoising & CNN with separable convolution, and gradient prior for image denoising \\
\hline Fu et al. (2018) 58 & CNN & Salt and pepper noise removal & CNN with non-local switching filter for salt and pepper noise \\
\hline Yeh et al. (2018) 243 & CNN & Image denoising super-resolution and inpainting & GAN with MAP for image restoration \\
\hline Liu et al. (2018) 136] & $\mathrm{CNN}$ & Medical image denoising, computed tomography perfusion for image denoising & CNN with genetic algorithm for medical image denoising \\
\hline Tassano et al. (2019) 202] & CNN & Gaussian image denoisng & CNN with noise level, upscaling, downscaling operation for image denoising \\
\hline Heckel et al. (2018) 76 & CNN & Image denoisng & CNN with deep prior for image denoising \\
\hline Jiao et al. (2017) [100] & CNN & Gaussian image denoisng, image inpainting & CNN with inference, residual operation for image restoration \\
\hline Wang et al. (2017) 218 & CNN & Image denoising & CNN with total variation for image denoising \\
\hline Li et al. (2019) [128] & CNN & Image painting & CNN with split Bregman iteration algorithm for image painting \\
\hline Sun et al. (2018) 195 & CNN & Gaussian image denoisng & GAN with skip-connections, and ResNet blocks for image denoising \\
\hline Zhi et al. (2018) 272 & $\mathrm{CNN}$ & Gaussian image denoising & GAN with multiscale for image denoising \\
\hline Du et al. (2018) 51] & CNN & Gaussian image denoising & CNN with wavelet for medcial image restoration \\
\hline Liu et al. (2019) [140] & CNN & Gaussian image denoising, real noisy image denoising, rain removal & Dual CNN with residual operations for image restoration \\
\hline Khan et al. (2019) 105 & CNN & Symbol denoising & CNN with quadrature amplitude modulation for symbol denoising \\
\hline Zhang et al. (2019) 266 & CNN & Image Possian denoising & CNN with variance-stabilizing transformation for poisson denoising \\
\hline Cruz et al. (2018) 43] & CNN & Gaussian image denoising & CNN with nonlocal filter for image denoising \\
\hline Jia et al. (2019) 95 & CNN & Gaussian image denoising & CNN based on a fractional-order differential equation for image denoising \\
\hline
\end{tabular}

For improving denoising performance, a CNN combined optimization method was used to make a noisy image smooth [76, 65, 100]. A CNN with total variation denoising reduced the effect of noise pixels [218]. Combining the Split Bregman iteration algorithm and CNN [128] can enhance pixels through image depth to obtain a latent clean image. A dual-stage CNN with feature matching can better recover the detailed information of the clean image, especially noisy images [195]. The GAN with the nearest neighbor algorithm was effective in filtering out noisy images from clean images [272]. A combined CNN used wavefront coding to enhance the pixels of latent clean images via the transform domain [51]. Other effective denoising methods are shown in [140, 105, 66]. Table 3 shows detailed information about the combination of the optimization methods and CNN/NN in AWNI denoising.

\subsection{Deep learning techniques for real noisy image denoising}

There are mainly two types of deep learning techniques for image denoising: single end-to-end $\mathrm{CNN}$ and the combination of prior knowledge and CNN.

For the first method, changing the network architecture is an effective way to remove the noise from the given real corrupted image. Multiscale knowledge is effective for image denoising. For example, a CNN consisting of convolution, ReLU and RL employed different phase features to enhance the expressive ability of the low-light image denoising model [201]. To overcome the blurry and false image artifacts, a dual U-Net with skip connection was proposed for computed tomography (CT) image reconstruction [73]. To address the problem of resource-constraints problem, Tian et al. [207] used a dual CNN with batch renormalization [88], RL and dilated convolutions to deal with real noisy images. Based on nature of light images, two CNNs utilized anisotropic parallax analysis to generate structural parallax information for real noisy images [32]. Using a CNN to resolve remote sensing [97] and medical images [107] under low-light conditions proved effective [99]. To extract more detailed information, recurrent connections were used to enhance the representative ability to deal with corrupted images in the real world [64, 269]. To deal with unknown real noisy images, a residual structure was utilized to facilitate low-frequency features, and then, an attention mechanism [206] could be applied to extract more potential features from channels [11]. To produce the noisy image, a technique used imitating cameral pipelines to construct the degradation model in order to filter the real noisy images [91]. To tackle the problem of unpairing noisy images, an unsupervised learning method embedded into the CNN proved effective in image 
denoising [44]. The self-consistent GAN [236] first used a CNN to estimate the noise of the given noisy image as a label, and then, applied another $\mathrm{CNN}$ and the obtained label to remove the noise for other noisy images. This concept has also been extended to general CNNs. The Noise2Inverse method used a $\mathrm{CNN}$ to predict the value of a noisy pixel, according to its surrounding noisy pixels [78]. The attention mechanism merged into a 3D self-supervised network can improve the efficiency of removing the noise from medical noisy images [123]. More detailed information about the above research is shown in Table 4.

Table 4: CNNs for real noisy image denoising.

\begin{tabular}{|c|c|c|c|}
\hline References & Methods & Applications & Key words (remarks) \\
\hline Tao et al. (2019) [201] & CNN & Real noisy image denoising, low-light image enhancement & CNN with ReLU, and RL for real noisy image denoising \\
\hline Chen et al. (2018) 31] & CNN & Real noisy image denoising, blind denoising & GAN for real noisy image denoising \\
\hline Han et al. (2018) 73 & CNN & CT image reconstruction & U-Net with skip connection for CT image reconstruction \\
\hline Chen et al. (2018) 32] & CNN & Real noisy image denoising & CNNs with anisotropic parallax analysis for real noisy image denoising \\
\hline Jian et al. (2018) 97 & CNN & Low-light remote sense image denoising & CNN for image denoising \\
\hline Khoroushadi et al. (2019) 107 & CNN & Medical image denoising, $\mathrm{CT}$ image denoising & CNN for image denoising \\
\hline Jiang et al. (2018) 99] & CNN & Low-light image enhancement & CNN with symmetric pathways for low-light image enhancement \\
\hline Godard et al. (2018) 64 & CNN & Real noisy image denoising & CNN with recurrent connections for real noisy image denoising \\
\hline Zhao et al. (2019) 269] & CNN & Real noisy image denoising & CNN with recurrent conncetions for real noisy image denoising \\
\hline Anwar et al. (2019) [1] & CNN & Real noisy image denoising & CNN with RL, attention mechanism for real noisy image denoising \\
\hline Jaroensri et al. (2019) $[220]$ & CNN & Real noisy image denoising & CNN for real noisy image denoising \\
\hline Green et al. (2018) 67 & $\mathrm{CNN}$ & CT image denoising, real noisy image denoising & $\mathrm{CNN}$ for real noisy image denoising \\
\hline Brooks et al. (2019) [24] & CNN & Real noisy image denoising & CNN with image processing pipeline for real noisy image denoising \\
\hline Tian et al. (2020) 207] & CNN & Gaussian image denoising and real noisy image denoising & CNN with BRN, RL and dilated convolutions for image denosing \\
\hline Tian et al. (2020) 206 & CNN & Gaussian image denoising, blind denoising and real noisy image denoising & CNN with attention mechanism and sparse method for image denoising \\
\hline Tian et al. (2020) 208] & CNN & Gaussian image denoising, blind denoising and real noisy image denoising & Two CNNs with sparse method for image denoising \\
\hline Cui et al. (2019) 44] & CNN & Positron emission tomography image denoising, real noisy image denoising & Unsupervised $\mathrm{CNN}$ for unpair real noisy image denoising \\
\hline Yan et al. (2019) [236] & CNN & Real noisy image denoising & Self-supervised GAN for unpair real noisy image denoising \\
\hline Broaddus et al. (2020) 23] & CNN & Blind denoising and real noisy image denoising & Self-supervised CNN for unpair fluorescence microscopy image denoising \\
\hline Li et al. (2020) 123 & CNN & CT noisy image denoising & Self-supervised CNN with attention mechanism for unpair CT image denoising \\
\hline Hendriksen et al. (2020) 78 & CNN & CT noisy image denoising & Self-supervised CNN for unpair CT image denoising \\
\hline Wu et al. (2020) 224] & CNN & CT noisy image denoising & Self-supervised CNN for unpair dynamic CT image denoising \\
\hline
\end{tabular}

The method combining CNN and prior knowledge can better deal with both speed and complex noise task in real noisy images. Zhang et al. [259] proposed using half quadratic splitting (HQS) and $\mathrm{CNN}$ to estimate the noise from the given real noisy image. Guo et al. [71] proposed a threephase denoising method. The first phase used a Gaussian noise and in-camera processing pipeline to synthesize noisy images. The synthetic and real noisy images were merged to better represent real noisy images. The second phase used a sub-network with asymmetric and total variation losses to estimate the noise of real noisy image. The third phase exploited the original noisy image and estimated noise to recover the latent clean image. To address the problem of unpaired noisy images, the combination of CNN and prior knowledge in a semi-supervised way was developed [157]. A hierarchical deep GAN (HD-GAN) first used a cluster algorithm to classify multiple categories of each patient's CT, then built a dataset by collecting the images in the same categories from different patients. Finally, the GAN was used to deal with the obtained dataset for image denoising and classification [40]. A similar method performed well in 3D mapping [185].

A CNN with channel prior knowledge was effective for low-light image enhancement [200]. Table 5 shows the detailed information about the above research. 
Table 5: CNNs for real noisy image denoising.

\begin{tabular}{|c|c|c|c|}
\hline References & Methods & Applications & Key words (remarks) \\
\hline Zhang et al. (2017) [259] & CNN & Real-noisy image denoising & CNN with HQS for real noisy image \\
\hline Guo et al. (2019) [71] & CNN & Real-noisy image denoising & CNN and cameral processing pipeline for real noisy image \\
\hline Tao et al. (2019) [200] & CNN & Low-light image enhancement & CNN with channel prior for low-light image enhancement \\
\hline Ma et al. (2018) [148] & CNN & Tomography image denoising & GAN with edge-prior for CT image denoising \\
\hline Yue et al. (2018) [251] & CNN & Real-noisy image denoising, blind denoising & CNN with variational inference for blind denoising and real-noisy image denoisng \\
\hline Song et al. (2019) [192] & CNN & Real noisy image denoising & CNN with dynamic residual dense block for real noisy image denoising \\
\hline Lin et al. (2019) [131] & CNN & Real noisy image denoising & GAN with attentive mechanism and noise domain for real noisy image denoising \\
\hline Meng et al. (2020) [157] & CNN & Real noisy image denoising & CNN with semi-supervised learning for medical noisy image denoising \\
\hline Shantia et al. (2015) 185$]$ & CNN & Real noisy image denoising & CNN with semi-supervised learning for 3D map \\
\hline Choi et al. (2019) 40] & CNN & Real noisy image denoising & CNN with semi-supervised learning for medical noisy image denoising \\
\hline
\end{tabular}

\subsection{Deep learning techniques for blind denoising}

In the real world, images are easily corrupted and noise is complex. Therefore, blind denoising techniques are important [142]. An FFDNet [260] used noise level and noise as the input of CNN to train a denoiser for unknown noisy images. Subsequently, several methods were proposed to solve the problem of blind denoising. An image device mechanism proposed by Kenzo et al. [90] utilized soft shrinkage to adjust the noise level for blind denoising. For unpaired noisy images, using CNNs to estimate noise proved effective [191]. Yang et al. [239] used known noise levels to train a denoiser, then utilized this denoiser to estimate the level of noise. To resolve the problem of random noise attenuation, a CNN with RL was used to filter complex noise [256, 188]. Changing the network architecture can improve the denoising performance for blind denoising. Majumdar et al. [151] proposed the use of an auto-encoder to tackle unknown noise. For mixed noise, cascaded CNNs were effective in removing the additive white Gaussian noise (AWGN) and impulse noise [4]. Table 6 displays more information about these denoising methods.

Table 6: Deep learning techniques for blind denoising.

\begin{tabular}{|c|c|c|c|}
\hline References & Methods & Applications & Key words (remarks) \\
\hline Zhang et al. (2018) 260] & CNN & Blind denoising & CNN with varying noise level for blind denoising \\
\hline Kenzo et al. (2018) 90] & $\mathrm{CNN}$ & Blind denoising & CNN with soft shrinkage for blind denoising \\
\hline Soltanayev et al. (2018) 191 & CNN & Blind denoising & CNN for unpaired noisy images \\
\hline Yang et al. (2017) 239] & $\mathrm{CNN}$ & Blind denoising & CNNs with RL for blind denoising \\
\hline Zhang et al. (2018) 256 & $\mathrm{CNN}$ & Blind denoising, random noise & CNN with RL for blind denoising \\
\hline Si et al. (2018) 188 & CNN & Blind denoising, random noise & CNN for image denoising \\
\hline Majumdar et al. (2018) 99 & NN & Blind denoising & Auto-encoder for blind denoising \\
\hline Abiko et al. (2019) 64 & $\mathrm{CNN}$ & Blind denoising, complex noisy image denoising & cascaded CNNs for blind denoising \\
\hline Cha et al. (2019) 239] & CNN & Blind denoising & GAN for blind image denoising \\
\hline Tian et al. (2020) 206] & CNN & Gaussian image denoising, blind denoising and real noisy image denoising & CNN with attention mechanism and sparse method for image denoising \\
\hline
\end{tabular}

\subsection{Deep learning techniques for hybrid noisy image denoising}

In the real world, captured images are affected by complex environments. Motivated by that, several researchers proposed hybrid-noisy-image denoising techniques. Li et al. [127] proposed the combination of CNN and warped guidance to resolve the questions of noise, blur and JPEG compression. Zhang et al. [261] used a model to deal with multiple degradations, such as noise, blur kernel and low-resolution image. To enhance the raw sensor data, Kokkinos et al. [108] presented a residual $\mathrm{CNN}$ with an iterative algorithm for image demosaicing and denoising. To 
handle arbitrary blur kernels, Zhang et al. [262] proposed to use cascaded deblurring and singleimage super-resolution (SISR) networks to recover plug-and-play super-resolution images. These hybrid noisy image denoising methods are presented in Table 7.

Table 7: Deep learning techniques for hybrid noisy image denoising.

\begin{tabular}{|c|c|c|c|}
\hline References & Methods & Applications & Key words (remarks) \\
\hline Li et al. (2018) [127 & CNN & Noise, blur kernel, JPEG compression & The combination of CNN and warped guidance for multiple degradations \\
\hline Zhang et al. (2018) [261 & CNN & Noise, blur kernel, low-resolution image & CNN for multiple degradations \\
\hline Kokkinos et al. (2019) [108 & CNN & Image demosaicking and denoising & Residual CNN with iterative algorithm for image demosaicking and denoising \\
\hline
\end{tabular}

It is noted that an image carries finite information, which is not beneficial in real-world applications. To address this problem, burst techniques were developed [226]. However, the burst image suffered from the effects of noise and camera shake, which increased the difficulty of implementing the actual task. Recently, there has been much interest in deep learning technologies for burst image denoising, where the noise is removed frame by frame [9]. Recurrent fully convolutional deep neural networks can filter the noise for all frames in a sequence of arbitrary length [64]. The combination of CNN and the kernel method can boost the denoising performance for burst noisy images [153, 158]. In terms of complex background noisy images, an attention mechanism combined the kernel and CNN to enhance the effect of key features for burst image denoising, which can accelerate the training speed [255]. For low-light conditions, using a CNN to map a given burst noisy image to sRGB outputs can obtain a multi-frame denoising image sequence [269]. To reduce network complexity, a CNN with residual learning directly trained a denoising model rather than an explicit aligning procedure [199]. These burst denoising methods are listed in Table 8.

Table 8: Deep learning techniques for burst denoising.

\begin{tabular}{|c|c|c|c|}
\hline References & Methods & Applications & Key words (remarks) \\
\hline Xia et al. (2019) [226] & CNN & Burst denoising & CNN for burst denoising \\
\hline Aittala et al. (2018) $[9]$ & CNN & Burst denoising & CNN for burst denoising \\
\hline Godard et al. (2018) [64 & CNN & Burst denoising & CNN for burst denoising \\
\hline Marin et al. (2019) [153] & CNN & Burst denoising & CNN with kernel idea for burst denoising \\
\hline Mildenhall et al. (2018) [158] & CNN & Burst denoising & CNN with kernel idea for burst denoising \\
\hline Zhang et al. (2020) [255 & CNN & Burst denoising & CNN with kernel idea and attention idea for burst denoising \\
\hline Zhao et al. (2019) [269] & CNN & Burst denoising & CNN for burst denoising \\
\hline Tan et al. (2019) 199 & CNN & Burst denoising & CNN without explicit aligning procedure for burst denoising \\
\hline
\end{tabular}

Similar to burst images, video detection is decomposed into each frame. Therefore, deep learning techniques for additive white noisy-image denoising, real noisy image denoising, blind denoising , hybrid noisy image denoising are also suitable to video denoising [182, 216]. A recurrent neural network [33] utilized an end-to-end CNN to remove the noise from corrupted video. To improve video denoising, reducing the video redundancy is an effective method. A non-local patch idea fused CNN can efficiently suppress the noise for video and image denoising [46]. A CNN combined temporal information to make a tradeoff between performance and training efficiency in video denoising [203]. For blind video denoising, a two-stage CNN proved to be a good choice [53]. The first phase trained a video denoising model by fine-tuning a pre-trained AWGN denoising network [53]. The second phase obtained latent clean video by the obtained video denoising model. These video denoising methods are described in Table 9. 
Table 9: Deep learning techniques for video denoising.

\begin{tabular}{|c|c|c|c|}
\hline References & Methods & Applications & Key words (remarks) \\
\hline Sadda et al. (2018) [182] & CNN & Medical noisy video & CNN for video denoising \\
\hline Wang et al. (2020) [216] & CNN & Additive white Gaussian and salt-and-pepper noisy video & CNN for video denoising \\
\hline Chen et al. (2016) [33] & CNN & Additive white Poisson-Gaussian noisy video & CNN with non-local idea for video denoising \\
\hline Davy et al. (2018) [46] & CNN & Additive white Gaussian noisy video & CNN with temporal information for video denoising \\
\hline Tassano et al. (2019) [203] & CNN & Additive white Gaussian noisy video & CNN with pre-trained technology for blind video denoising \\
\hline Ehret et al. (2019) [53] & CNN & blind video denoising &
\end{tabular}

\section{Experimental results}

\subsection{Datasets}

\subsubsection{Training datasets}

The training datasets are divided into two categories: gray-noisy and color-noisy images. Gary-noisy image datasets can be used to train Gaussian denoisers and blind denoisers. They included the BSD400 dataset [21] and Waterloo Exploration Database [147]. The BSD400 dataset was composed of 400 images in .png format, and was cropped into a size of $180 \times 180$ for training a denoising model. The Waterloo Exploration Database consisted of 4,744 nature images with a .png format. Color-noisy images included the BSD432 [258], Waterloo Exploration Database and polyU-Real-World-Noisy-Images datasets [230]. Specifically, the polyU-Real-World-NoisyImages consisted of 100 real noisy images with sizes of $2,784 \times 1,856$ obtained by five cameras: a Nikon D800, Canon 5D Mark II, Sony A7 II, Canon 80D and Canon 600D.

\subsubsection{Test datasets}

The test datasets included gray-noisy and color-noisy image datasets. The gray-noisy image dataset was composed of Set12 and BSD68 [258]. The Set12 contained 12 scenes. The BSD68 contained 68 nature images. They were used to test the Gaussian denoiser and a denoiser of blind noise. The color-noisy image dataset included CBSD68, Kodak24 [57], McMaster [263], cc [160], DND [171], NC12 [112], SIDD [3] and Nam [160]. The Kodak24 and McMaster contained 24 and 18 color noisy images, respectively. The cc contained 15 real noisy images of different ISO, i.e., 1,600, 3,200 and 6,400. The DND contained 50 real noisy images and the clean images were captured by low-ISO images. The NC12 contained 12 noisy images and did not have ground-truth clean images. The SIDD contained real noisy images from smart phones, and consisted of 320 image pairs of noisy and ground-truth images. The Nam included 11 scenes, which were saved in JPGE format.

\subsection{Experimental results}

To verify the denoising performance of some methods mentaioned in Section 3, we conducted some experiments on the Set12, BSD68, CBSD68, Kodak24, McMaster, DND, SIDD, Nam, cc and $\mathrm{NC} 12$ datasets in terms of quantitative and qualitative evaluations. The quantitative evaluation mainly used peak-signal-to-noise-ratio (PSNR) [84] values of different denoisers to test the denoising effects. Additionally, we used the runtime of denoising of an image to support the PSNR for quantitative evaluation. The qualitative evaluation used visual figures to show the recovered clean images. 
Table 10: PSNR (dB) of different methods on the BSD68 for different noise levels (i.e., 15, 25 and 50).

\begin{tabular}{|c|c|c|c|}
\hline Methods & 15 & 25 & 50 \\
\hline BM3D [45] & 31.07 & 28.57 & 25.62 \\
\hline WNNM 69 & 31.37 & 28.83 & 25.87 \\
\hline EPLL [274 & 31.21 & 28.68 & 25.67 \\
\hline MLP [25] & - & 28.96 & 26.03 \\
\hline CSF 184 & 31.24 & 28.74 & - \\
\hline TNRD [35] & 31.42 & 28.92 & 25.97 \\
\hline ECNDNet 204 & 31.71 & 29.22 & 26.23 \\
\hline RED 152 & - & - & 26.35 \\
\hline DnCNN 258 & 31.72 & 29.23 & 26.23 \\
\hline DDRN [219] & 31.68 & 29.18 & 26.21 \\
\hline PHGMS [13] & 31.86 & - & 26.36 \\
\hline MemNet [197 & - & - & 26.35 \\
\hline EEDN [34] & 31.58 & 28.97 & 26.03 \\
\hline NBCNN 213] & 31.57 & 29.11 & 26.16 \\
\hline NNC 253 & 31.49 & 28.88 & 25.25 \\
\hline ELDRN [165 & 32.11 & 29.68 & 26.76 \\
\hline PSN-K [10] & 31.70 & 29.27 & 26.32 \\
\hline PSN-U [10] & 31.60 & 29.17 & 26.30 \\
\hline DDFN [42] & 31.66 & 29.16 & 26.19 \\
\hline CIMM [12] & 31.81 & 29.34 & 26.40 \\
\hline DWDN [122] & 31.78 & 29.36 & - \\
\hline MWCNN 137 & 31.86 & 29.41 & 26.53 \\
\hline BM3D-Net 237 & 31.42 & 28.83 & 25.73 \\
\hline MPFE-CNN [103] & 31.79 & 29.31 & 26.34 \\
\hline IRCNN 259] & 31.63 & 29.15 & 26.19 \\
\hline FFDNet [260 & 31.62 & 29.19 & 26.30 \\
\hline BRDNet 207 & 31.79 & 29.29 & 26.36 \\
\hline ETN 218 & 31.82 & 29.34 & 26.32 \\
\hline ADNet 206 & 31.74 & 29.25 & 26.29 \\
\hline NN3D 43 & - & - & 26.42 \\
\hline FOCNet 95 & 31.83 & 29.38 & 26.50 \\
\hline DudeNet 208] & 31.78 & 29.29 & 26.31 \\
\hline
\end{tabular}

Table 11: FSIM of different methods on the BSD68 for different noise levels (i.e., 15, 25 and 50).

\begin{tabular}{|c|c|c|c|}
\hline Methods & 15 & 25 & 50 \\
\hline BM3D [45] & 0.9894 & 0.9811 & 0.9629 \\
\hline MLP [25] & 0.9671 & 0.9821 & 0.9344 \\
\hline TNRD [35] & 0.9697 & 0.9820 & 0.9291 \\
\hline ECNDNet [204] & 0.9911 & 0.9837 & 0.9686 \\
\hline IRCNN [259] & 0.9905 & 0.9835 & 0.9700 \\
\hline BRDNet [207] & 0.9913 & 0.9841 & 0.9687 \\
\hline ADNet [206] & 0.9912 & 0.9837 & 0.9673 \\
\hline
\end{tabular}

\subsubsection{Deep learning techniques for additive white noisy-image denoising}

Comparisons of denoising methods should take into consideration additive white noise, including Gaussian, Poisson, low-light noise, and salt and pepper noise, all of which have significantly different noise levels. Furthermore, many of the methods use different tools, which can have a significant influence on denoising results. For these reasons, we chose typical Gaussian noise to test the denoising performance of the various methods. In addition, most of the denoising methods use PSNR as a quantitative index. Therefore, we used the BSD68, Set12, CBSD68, Kodak24 and McMaster datasets to test the denoising performance of deep learning techniques for additive white noisy-image denoising. Table 10 shows the PSNR values of different networks with different noise levels for gray additive white noisy image denoising. To understand the denoising performance 
of different methods, we used a feature similarity index (FSIM) [264] as a visual quality metric to conduct experiments on BSD68 for different noise levels (i.e., 15, 25 and 50), as shown in Table 11. To test the ability of dealing with single gray additive white noisy images from different networks, Set12 was used to conduct experiments, as shown in Table 12. Table 13 displays the denoising performance of different methods for color additive white noisy image denoising. Table 14 presents the efficiency of different methods for image denoising. For qualitative analysis, we magnified one area of the latent clean image from different methods. As shown in Figs. 7-10, the observed area is clearer, and the corresponding method has better denoising performance.

\subsubsection{Deep learning techniques for real-noisy image denoising}

For testing the denoising performance of deep learning techniques for real-noisy images, the public datasets, such as DND, SIDD, Nam and CC, were chosen to design the experiments. We chose not to use the NC12 dataset because the ground-truth clean images from NC12 were unavailable. Also, to help readers better understand these methods, we added several traditional denoising methods, such as BM3D, as comparative methods. From Tables 15 and 16, we can see that the DRDN obtained the best results on the DND and SSID in real-noisy image denoising, respectively. For compressed noisy images, the AGAN obtained excellent performance, as shown in Table 17. For real noisy images of different ISO values, the SDNet and BRDNet achieved the best and second-best denoising performance, respectively, as described in Table 18.

\subsubsection{Deep learning techniques for blind denoising}

It is known that noise is complex in the real world, and not subject to rules. This is why blind denoising techniques, especially deep learning techniques, have been developed. Comparing the denoising performance of different deep learning techniques is very useful. The state-of-the-art denoising methods such as DnCNN, FFDNet, ADNet, SCNN and G2G1 on the BSD68 and Set12 were chosen to design the experiments. FFDNet and ADNet are superior to other methods in blind denoising, as shown in Tables 19 and 20, respectively.

\subsubsection{Deep learning techniques for hybrid-noisy-image denoising}

In the real world, corrupted images may include different kinds of noise [74], which makes it very difficult to recover a latent clean image. To resolve this problem, deep learning techniques based multi-degradation idea have been proposed, as discussed in Section 3.4. Here we introduce the denoising performance of the multi-degradation model, as shown in Table 21, where the WarpNet method is shown to be very competitive in comparison with other popular denoising methods, such as DnCNN and MemNet.

\section{Discussion}

Deep learning techniques are seeing increasing use in image denoising. This paper offers a survey of these techniques in order to help readers understand these methods. In this section, we present the potential areas of further research for image denoising and points out several as yet unsolved problems. 
Table 12: PSNR (dB) of different methods on the Set12 for different noise levels (i.e., 15, 25 and 50).

\begin{tabular}{|c|c|c|c|c|c|c|c|c|c|c|c|c|c|}
\hline Images & $\operatorname{man}$ & ouse & ppers & arfish & onarch & rplane & Parrot & Lena & arbara & Boat & Man & Couple & ver \\
\hline Noise Level & \multicolumn{13}{|c|}{$\sigma=15$} \\
\hline BM3D [45] & .91 & .93 & 32.69 & 31.1 & 31.8 & 31.07 & 31.37 & 34.26 & 33. & 32.13 & 31.92 & 2.10 & 32.37 \\
\hline WNNM [69] & .17 & .13 & 99 & .82 & 32.71 & .39 & 31.62 & 34.27 & 33.60 & 32.27 & 32.11 & 32.17 & 32.70 \\
\hline EPLL [274] & 85 & .17 & 64 & 1.13 & 32.10 & .19 & 31.42 & 3.92 & 31.38 & 1.93 & 32.00 & 1.93 & 2.14 \\
\hline CSF [184] & 95 & .39 & 85 & .55 & 2.33 & 33 & 31.37 & 4.06 & 31.92 & 2.01 & 32.08 & 31.98 & 2.32 \\
\hline TNRD 35 & 19 & .53 & 04 & .75 & 32.56 & .46 & 31.63 & 34.24 & 32.13 & 2.14 & 32.23 & 2.11 & 2.50 \\
\hline CNDNet [204] & 56 & 97 & 25 & 32.17 & 3.11 & 70 & 31.82 & 34.52 & 32.41 & 32.37 & 32.39 & 2.39 & 2.81 \\
\hline DnCNN 258 & 61 & .97 & 30 & 32.20 & 33.09 & .70 & 31.83 & 34.62 & 32.64 & 32.42 & 32.46 & 2.47 & 2.86 \\
\hline PSN-K $[10]$ & .58 & 35.04 & 23 & 32.17 & 11 & .75 & 31.89 & 34.62 & 32.64 & 32.52 & 32.39 & 32.43 & 2.86 \\
\hline PSN-U [10] & .04 & .03 & .21 & 31.94 & 2.93 & .61 & 31.62 & 34.56 & 32.49 & 32.41 & 32.37 & 32.43 & 32.72 \\
\hline CIMM $[12$ & 2.61 & 35.21 & .21 & 32.35 & 33 & .77 & 32.01 & 34.69 & 32.74 & 32.44 & 32.50 & 32.52 & 32.95 \\
\hline IRCNN [259] & 2.55 & 34.89 & 31 & 32.02 & 2.82 & 31.70 & 31.84 & 34.53 & 32.43 & 32.34 & 32.40 & 32.40 & 32.77 \\
\hline FFDNet [260] & .43 & 35.07 & 25 & 31.99 & 2.66 & 31.57 & 31.81 & 34.62 & 32.54 & 32.38 & 32.41 & 32.46 & 32.77 \\
\hline BRDNet [207] & 80 & 35.27 & 47 & 32.24 & .35 & 31.85 & 32.00 & 34.75 & 32.93 & 2.55 & 32.50 & 32.62 & 33.03 \\
\hline ADNet [206 & 81 & 35.22 & .49 & 32.17 & .17 & 31.86 & 31.96 & 34.71 & 32.80 & 32.57 & 32.47 & 32.58 & 32.98 \\
\hline DudeNet [208] & .71 & 35.13 & 38 & 32.29 & 3.28 & 31.78 & 31.93 & 34.66 & 32.73 & 32.46 & 32.46 & 32.49 & 32.94 \\
\hline Noise Level & \multicolumn{13}{|c|}{$=25$} \\
\hline BM3D 45 & 45 & 32.85 & 16 & 28.56 & 29.25 & 28.42 & \begin{tabular}{|l|}
28.93 \\
\end{tabular} & 32.07 & 30.71 & 29.90 & 29.61 & 29.71 & 29.97 \\
\hline WNNM 69 & .64 & 33.22 & 30.42 & 29.03 & 9.84 & 28.69 & 29.15 & 32.24 & 31.24 & 30.03 & 9.76 & 29.82 & 30.26 \\
\hline EPLL $[274$ & 26 & 32.17 & 30 & 28.51 & 9.39 & .61 & 28.95 & 31.73 & 28.61 & 29.74 & 29.66 & 9.53 & 29.69 \\
\hline MLP [25] & 61 & 56 & 3 & .82 & 29.61 & .82 & 29.25 & 32.25 & 9.54 & 9.97 & 29.88 & 9.73 & 0.03 \\
\hline $\mathrm{F}[184]$ & 48 & .39 & & .80 & 29.62 & .72 & 28.90 & 31.79 & 9.03 & 9.76 & 29.71 & 9.53 & 9.84 \\
\hline TNRD & 72 & 3 & & .02 & & 88 & 29.18 & 32.00 & 29.41 & 9.91 & 29.87 & 1 & 0.06 \\
\hline ECNDN & & J. & & 29.43 & & .07 & 29.38 & 32.38 & 29.84 & 30.14 & 30.03 & 0.03 & 0.39 \\
\hline DnCNI & 18 & 33.06 & 87 & .41 & 30.28 & 13 & 29.43 & 32.44 & 30.00 & 30.21 & 30.10 & 30.12 & 30.43 \\
\hline PSN- & .28 & 33.26 & & .57 & 30.30 & .28 & 29.38 & 32.57 & 30.17 & 30.31 & 30.10 & 30.18 & 30.53 \\
\hline $\mathrm{Ps}$ & 79 & 23 & & .30 & 30.17 & .06 & 29.25 & 32.45 & 29.94 & 30.25 & 30.05 & 30.12 & 30.38 \\
\hline & & 44 & & .77 & 30.62 & 23 & 29.61 & 32.66 & 30.29 & 30.30 & 30.18 & 30.24 & 30.62 \\
\hline IRCNN & & 06 & & 27 & 30.09 & 12 & 29.47 & 32.43 & 29.92 & 30.17 & 30.04 & 30.08 & 30.38 \\
\hline $\mathrm{FH}$ & .10 & 33.28 & & 29.32 & 30. & .04 & 29.44 & 32.57 & 30.01 & 30.25 & 30.11 & 30.20 & 30.44 \\
\hline BRDNet [207] & 39 & 33.41 & 4 & 29.46 & 2050 & .20 & 29.55 & 32.65 & 30.34 & 30.33 & 30.14 & 30.28 & 30.61 \\
\hline ADNet 206 & .34 & 33.41 & & 29.41 & & 17 & 29.49 & 32.61 & 30.25 & 30.37 & 30.08 & 30.24 & 30.58 \\
\hline DudeNet [208] & & 33.24 & & $\sqrt{3}$ & & .14 & \begin{tabular}{|l|l|}
29.48 \\
\end{tabular} & 2.52 & 0.15 & 30.24 & 30.08 & 30.15 & 30.52 \\
\hline Noise Level & \multicolumn{13}{|c|}{$=50$} \\
\hline BM3D 45 & 13 & 29.69 & o & 25.04 & 5.82 & 25.10 & 25.90 & 29.05 & 27.22 & 26.78 & 26.81 & 26.46 & 26.72 \\
\hline WNNM 69 & 26.45 & 30.33 & & 25.44 & & 25.42 & 26.14 & 29.25 & 27.79 & 26.97 & 26.94 & 26.64 & 27.05 \\
\hline EPLL 274] & 10 & 29.12 & & 25.12 & 25.94 & .31 & 25.95 & 28.68 & 24.83 & 26.74 & 26.79 & 26.30 & 26.47 \\
\hline MLP [25] & & 29.64 & & 2 & & 56 & 26.12 & 29.32 & 25.24 & 27.03 & 27.06 & 26.67 & 26.78 \\
\hline & & 29.48 & & 25.42 & & 59 & 26.16 & 28.93 & 25.70 & 26.94 & 6.98 & 26.50 & 26.81 \\
\hline ECNDNet [204] & 07 & 30.12 & & 72 & 26.8 & 79 & 26.32 & 29.29 & 26.26 & 7.16 & 27.11 & 26.84 & 27.15 \\
\hline DnCNN [258] & & 30.00 & & & 2 & 87 & 26.48 & 29.39 & 26.22 & 27.20 & 27.24 & 26.90 & 27.18 \\
\hline & & 30.34 & & & & & 26.56 & 29.54 & 26.45 & 7.20 & 27.21 & 27.09 & 87.30 \\
\hline 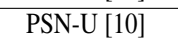 & & 30.21 & & & & & 26.62 & 29.54 & 26.56 & 27.27 & 27.23 & 27.04 & 27.31 \\
\hline CIMM [12] & 25 & 30.70 & & 26.05 & 27. & 26.06 & 26.53 & 29.65 & 26.62 & 27.36 & 27.26 & 27.24 & 27.46 \\
\hline IRCNN 259 & 88 & 29.96 & & 25.57 & 26.6 & .89 & 26.55 & 29.40 & 26.24 & 27.17 & 27.17 & 26.88 & 27.14 \\
\hline FFDNet [260] & 7.05 & 30.37 & & 25.75 & 26.81 & 25.89 & 26.57 & 29.66 & 26.45 & 27.33 & 27.29 & 27.08 & 27.32 \\
\hline BRDNet [207] & .44 & 30.53 & 27.01 & 25.77 & 26.97 & 25.93 & 26.66 & 29.73 & 26.85 & 27.38 & 27.27 & 27.17 & 27.45 \\
\hline ADNet 206 & 27.31 & 30.59 & 27.69 & 25.70 & 26.90 & 25.88 & 26.56 & 29.59 & 26.64 & 27.35 & 27.17 & 27.07 & 27.37 \\
\hline DudeNet [208 & 27.22 & 30.27 & 27.51 & 25.88 & 26.93 & 25.88 & 26.50 & 29.45 & 26.49 & 27.26 & 27.19 & 26.97 & 27.30 \\
\hline
\end{tabular}

Image denoising based on deep learning techniques mainly are effective in increasing denoising performance and efficiency, and performing complex denoising tasks. Solutions for improving denoising performance include the following:

1) Enlarging the receptive field can capture more context information. Enlarging the receptive 
Table 13: PSNR (dB) of different methods on the CBSD68, Kodak24 and McMaster for different noise levels (i.e., $15,25,35,50$ and 75$)$.

\begin{tabular}{|c|c|c|c|c|c|c|}
\hline Datasets & Methods & $\sigma=15$ & $\sigma=25$ & $\sigma=35$ & $\sigma=50$ & $\sigma=75$ \\
\hline \multirow{15}{*}{ CBSD68 } & CBM3D [45] & 33.52 & 30.71 & 28.89 & 27.38 & 25.74 \\
\hline & DnCNN [258] & 33.98 & 31.31 & 29.65 & 28.01 & - \\
\hline & DDRN [219] & 33.93 & 31.24 & - & 27.86 & - \\
\hline & EEDN [34] & 33.65 & 31.03 & - & 27.85 & - \\
\hline & DDFN [42] & 34.17 & 31.52 & 29.88 & 28.26 & - \\
\hline & CIMM [12] & 31.81 & 29.34 & - & 26.40 & - \\
\hline & BM3D-Net [237] & 33.79 & 30.79 & - & 27.48 & - \\
\hline & IRCNN [259] & 33.86 & 31.16 & 29.50 & 27.86 & - \\
\hline & FFDNet [260] & 33.80 & 31.18 & 29.57 & 27.96 & 26.24 \\
\hline & BRDNet [207] & 34.10 & 31.43 & 29.77 & 28.16 & 26.43 \\
\hline & GPADCNN [39] & 33.83 & 31.12 & 29.46 & - & 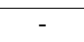 \\
\hline & FFDNet [202] & 33.76 & 31.18 & 29.58 & - & 26.57 \\
\hline & ETN $[218$ & 34.10 & 31.41 & - & 28.01 & - \\
\hline & ADNet [206] & 33.99 & 31.31 & 29.66 & 28.04 & 26.33 \\
\hline & DudeNet [208] & 34.01 & 31.34 & 29.71 & 28.09 & 26.40 \\
\hline \multirow{8}{*}{ Kodak24 } & CBM3D [45] & 34.28 & 31.68 & 29.90 & 28.46 & 26.82 \\
\hline & DnCNN 258 & 34.73 & 32.23 & 30.64 & 29.02 & - \\
\hline & IRCNN 259 & 34.56 & 32.03 & 30.43 & 28.81 & - \\
\hline & FFDNet [260] & 34.55 & 32.11 & 30.56 & 28.99 & 27.25 \\
\hline & BRDNet [207] & 34.88 & 32.41 & 30.80 & 29.22 & 27.49 \\
\hline & FFDNet [202] & 34.53 & 32.12 & 30.59 & - & 27.61 \\
\hline & ADNet [206] & 34.76 & 32.26 & 30.68 & 29.10 & 27.40 \\
\hline & DudeNet [208 & 34.81 & 32.26 & 30.69 & 29.10 & 27.39 \\
\hline \multirow{6}{*}{ McMaster } & CBM3D 45] & 34.06 & 31.66 & 29.92 & 28.51 & 26.79 \\
\hline & DnCNN 258 & 34.80 & 32.47 & 30.91 & 29.21 & - \\
\hline & IRCNN 259 & 34.58 & 32.18 & 30.59 & 28.91 & - \\
\hline & FFDNet [260] & 34.47 & 32.25 & 30.76 & 29.14 & 27.29 \\
\hline & BRDNet 207 & 35.08 & 32.75 & 31.15 & 29.52 & 27.72 \\
\hline & ADNet [206] & 34.93 & 32.56 & 31.00 & 29.36 & 27.53 \\
\hline
\end{tabular}

Table 14: Running time of 13 popular denoising methods for the noisy images of sizes $256 \times 256,512 \times 512$ and $1024 \times 1024$.

\begin{tabular}{|c|c|c|c|c|}
\hline Methods & Device & $256 \times 256$ & $512 \times 512$ & $1024 \times 1024$ \\
\hline BM3D [45] & CPU & 0.65 & 2.85 & 11.89 \\
\hline WNNM [69] & CPU & 203.1 & 773.2 & 2536.4 \\
\hline EPLL [274] & CPU & 25.4 & 45.5 & 422.1 \\
\hline MLP [25] & CPU & 1.42 & 5.51 & 19.4 \\
\hline CSF [184] & CPU & 2.11 & 5.67 & 40.8 \\
\hline CSF [184] & GPU & - & 0.92 & 1.72 \\
\hline TNRD [35] & CPU & 0.45 & 1.33 & 4.61 \\
\hline TNRD [35] & GPU & 0.010 & 0.032 & 0.116 \\
\hline ECNDNet [204] & GPU & 0.012 & 0.079 & 0.205 \\
\hline DnCNN [258] & CPU & 0.74 & 3.41 & 12.1 \\
\hline DnCNN [258] & GPU & 0.014 & 0.051 & 0.200 \\
\hline FFDNet [260] & CPU & 0.90 & 4.11 & 14.1 \\
\hline FFDNet [260] & GPU & 0.016 & 0.060 & 0.235 \\
\hline IRCNN [259] & CPU & 0.310 & 1.24 & 4.65 \\
\hline IRCNN [259] & GPU & 0.012 & 0.038 & 0.146 \\
\hline BRDNet [207] & GPU & 0.062 & 0.207 & 0.788 \\
\hline ADNet [206] & GPU & 0.0467 & 0.0798 & 0.2077 \\
\hline DudeNet [208] & GPU & 0.018 & 0.422 & 1.246 \\
\hline
\end{tabular}




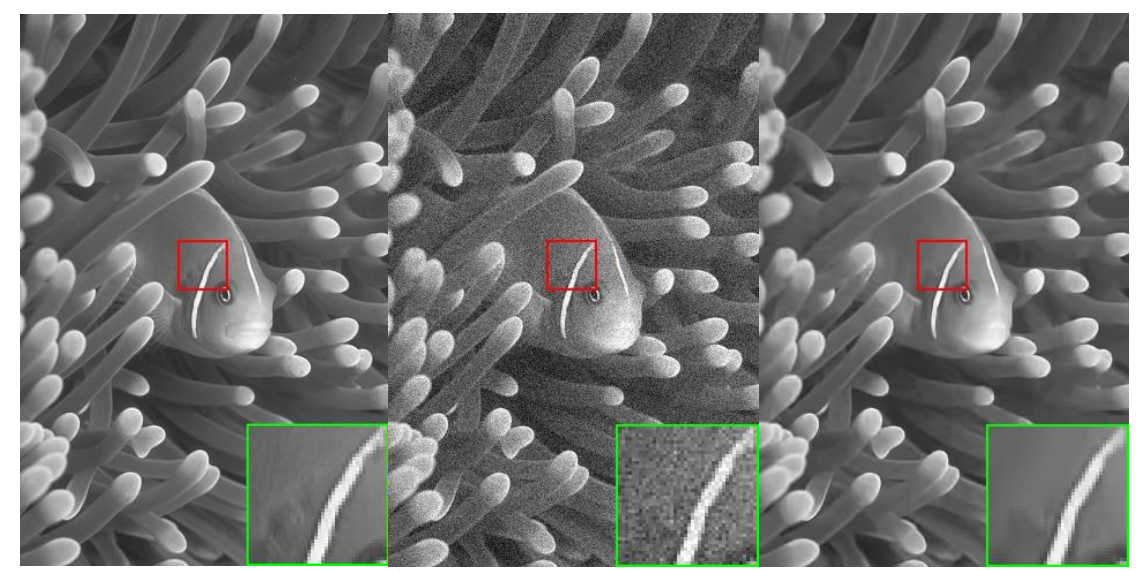

(a)

(b)

(c)

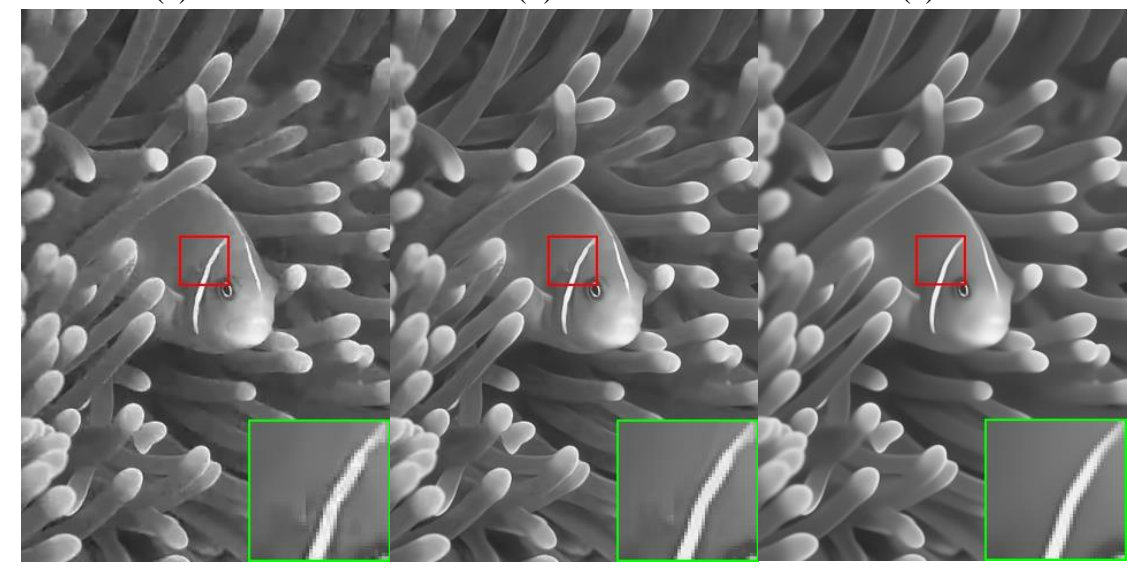

(d)

(e)

(f)

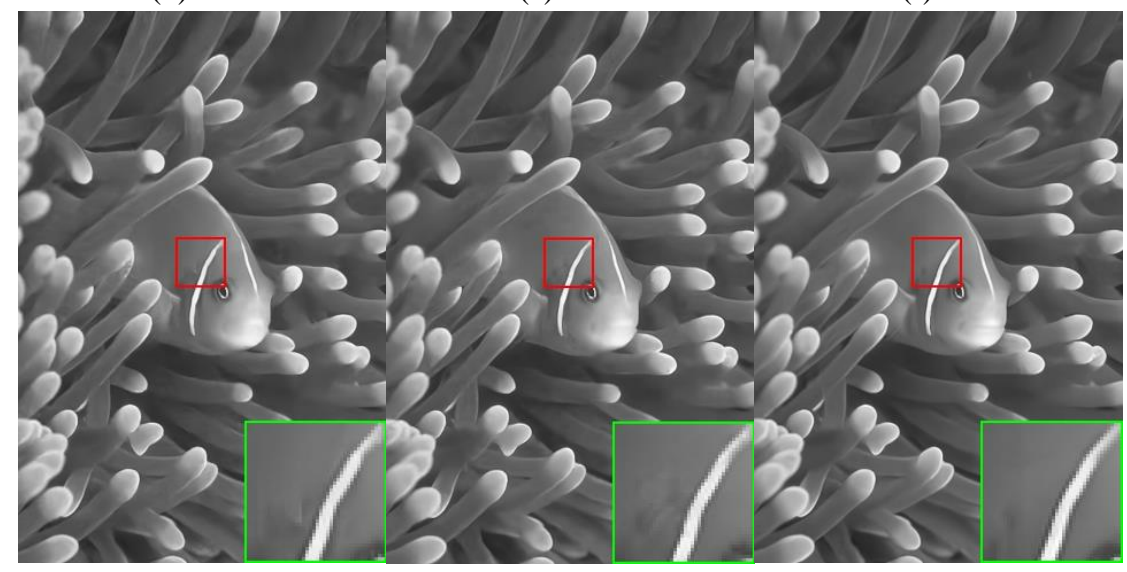

(g)

(h)

(i)

Figure 7: Denoising results of different methods on one image from the BSD68 with $\sigma=15$ : (a) original image, (b) noisy image/24.62dB, (c) BM3D/35.29dB, (d) EPLL/34.98dB, (e) DnCNN/36.20dB, (f) FFDNet/36.75dB, (g) IRCNN/35.94dB, (h) ECNDNet/36.03dB, and (i) BRDNet/36.59dB.

field can be accomplished by increasing the depth and width of the networks. However, this results in higher computational costs and more memory consumption. One technique for resolving this 


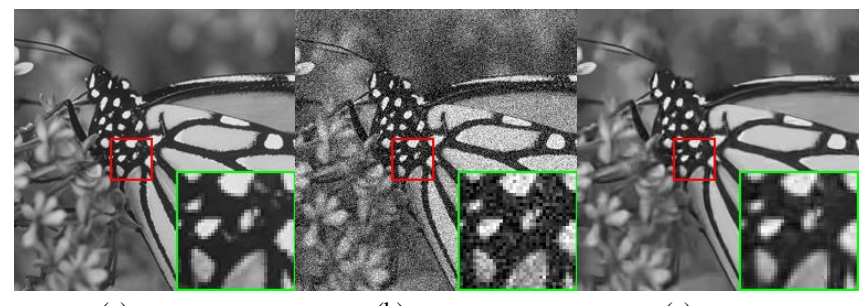

(a)

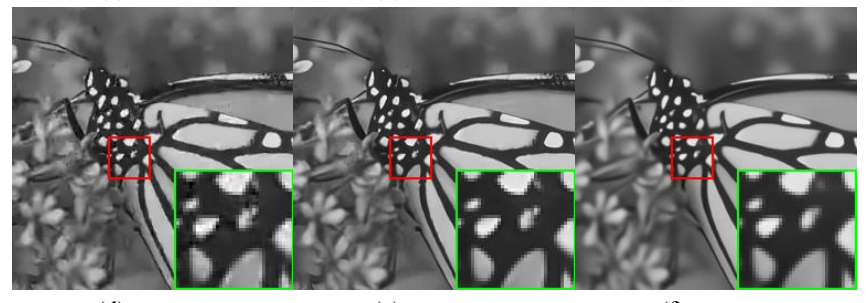

(d)

(e)

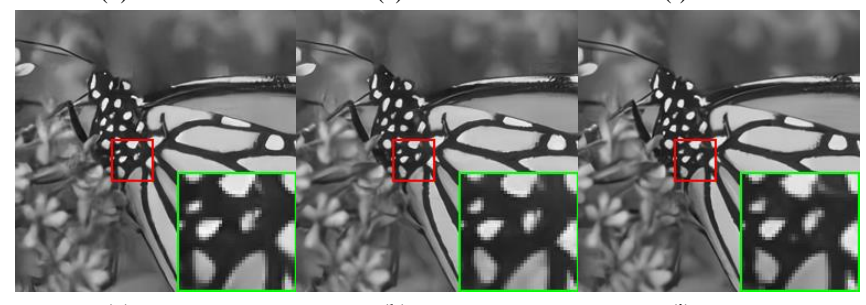

$(\mathrm{g})$

(h)

(i)

Figure 8: Denoising results of different methods on one image from the Set12 with $\sigma=25$ : (a) original image, (b) noisy image $/ 20.22 \mathrm{~dB}$, (c) BM3D/29.26dB, (d) EPLL/29.44dB, (e) DnCNN/30.28dB, (f) FFDNet/30.08dB, (g) IR$\mathrm{CNN} / 30.09 \mathrm{~dB}$, (h) ECNDNet/30.30dB, and (i) BRDNet/30.50dB.
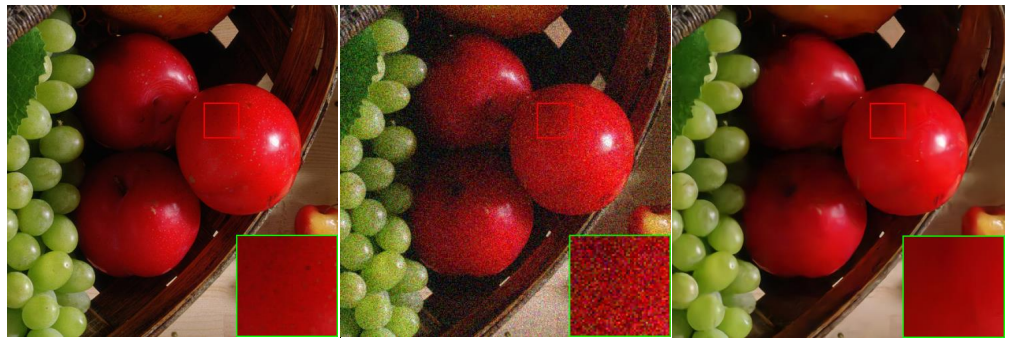

(a)

(b)

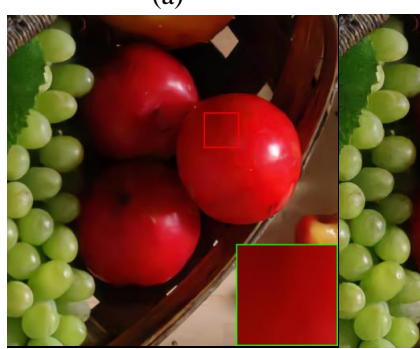

(d)

(c)

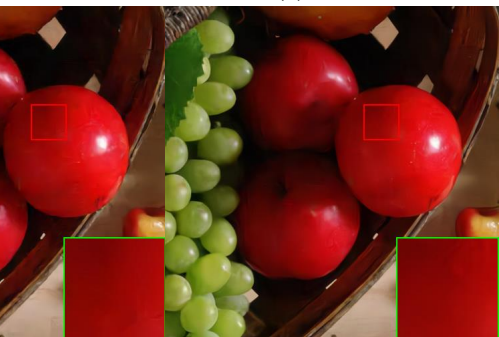

(e)

(f)

Figure 9: Denoising results of different methods on one image from the McMaster with $\sigma=35$ : (a) original image, (b) noisy image/18.46dB, (c) DnCNN/33.05B, (d) FFDNet/33.03dB, (e) IRCNN/32.74dB, and (f) BRDNet/33.26dB. 


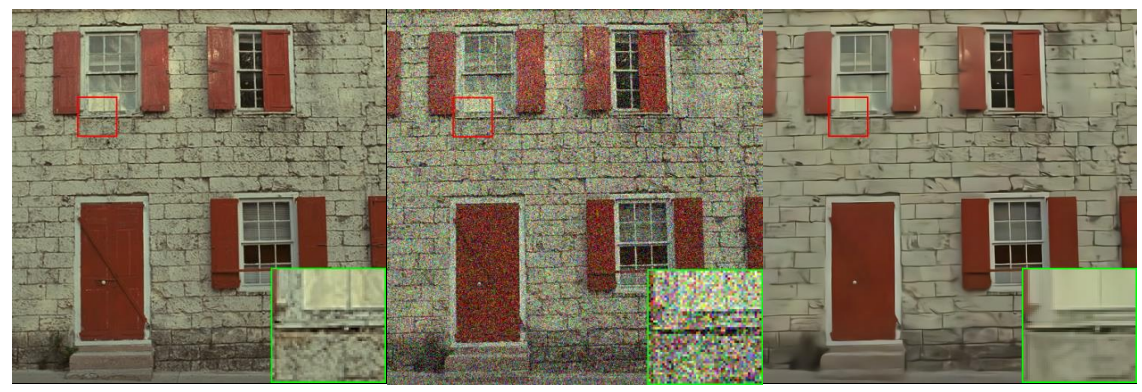

(a)

(b)

(c)

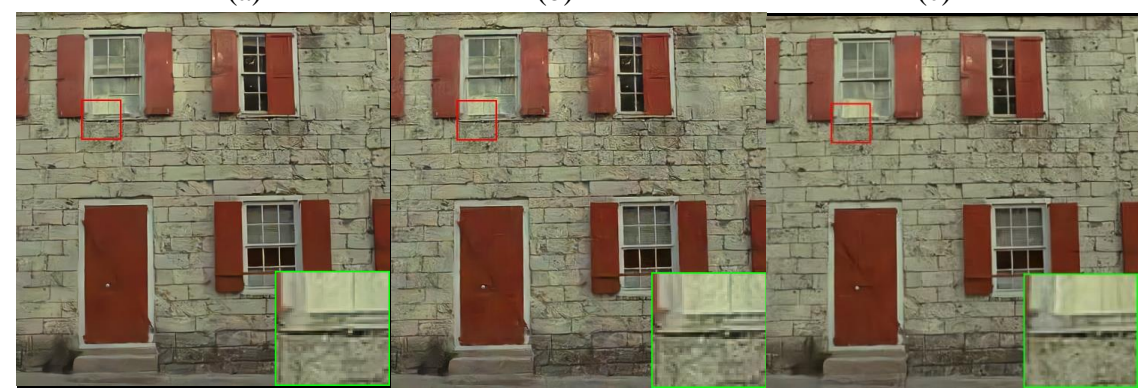

(d)

(e)

(f)

Figure 10: Denoising results of different methods on one image from the Kodak24 with $\sigma=50$ : (a) original image, (b) noisy image/14.58dB, (c) DnCNN/25.80B, (d) FFDNet/26.13dB, (e) IRCNN/26.10dB, and (f) BRDNet/26.33dB.

Table 15: PSNR (dB) of different methods on the DND for real-noisy image denoising.

\begin{tabular}{|c|c|}
\hline Methods & DND \\
\hline EPLL [274] & 33.51 \\
\hline TNRD [35] & 33.65 \\
\hline NCSR [49] & 34.05 \\
\hline MLP [25] & 34.23 \\
\hline BM3D [45] & 34.51 \\
\hline FoE [181] & 34.62 \\
\hline WNNM [69] & 34.67 \\
\hline KSVD [6] & 36.49 \\
\hline CDnCNN-B [258] & 32.43 \\
\hline FFDNet [260] & 34.40 \\
\hline MCWNNM [137] & 37.38 \\
\hline TWSC [232] & 37.94 \\
\hline GCBD [31] & 35.58 \\
\hline CIMM [12] & 36.04 \\
\hline CBDNet [71] & 37.72 \\
\hline VDN [251] & 39.38 \\
\hline DRDN [192] & 39.40 \\
\hline AGAN [131] & 38.13 \\
\hline
\end{tabular}

problem, is dilated convolution, which not only contributions to higher performance and efficiency, but is also very effective for mining more edge information.

2) The simultaneous use of extra information (also called prior knowledge) and a CNN is an effective approach to facilitate obtaining more accurate features. This is implemented by designing the loss function. 
Table 16: PSNR (dB) of different methods on the SIDD for real-noisy image denoising.

\begin{tabular}{|c|c|}
\hline Methods & SIDD \\
\hline CBM3D [45] & 25.65 \\
\hline WNNM [69] & 25.78 \\
\hline MLP [25] & 24.71 \\
\hline DnCNN-B [258] & 23.66 \\
\hline CBDNet [71] & 33.28 \\
\hline VDN [251] & 39.23 \\
\hline DRDN [192] & 39.60 \\
\hline
\end{tabular}

Table 17: PSNR (dB) of different methods on the Nam for real-noisy image denoising.

\begin{tabular}{|c|c|}
\hline Methods & Nam \\
\hline NI [5] & 31.52 \\
\hline TWSC [232] & 37.52 \\
\hline BM3D [45] & 39.84 \\
\hline NC [112] & 40.41 \\
\hline WNNM [69] & 41.04 \\
\hline CDnCNN-B [258] & 37.49 \\
\hline MCWNNM [137] & 37.91 \\
\hline CBDNet [71] & 41.02 \\
\hline CBDNet(JPEG) [71] & 41.31 \\
\hline DRDN [192] & 38.45 \\
\hline AGAN [131] & 41.38 \\
\hline
\end{tabular}

Table 18: PSNR (dB) of different methods on the cc for real-noisy image denoising.

\begin{tabular}{|c|c|c|c|c|c|c|c|c|c|c|c|}
\hline Camera Settings & CBM3D 45] & MLP 25] & TNRD 35 & DnCNN 258] & NI 5 & $\mathrm{NC}[112]$ & WNNM 69] & BRDNet 207] & SDNet [270] & ADNet 206 & DudeNet 208] \\
\hline \multirow{3}{*}{ Canon 5D ISO=3200 } & 39.76 & 39.00 & 39.51 & 37.26 & 35.68 & 38.76 & 37.51 & 37.63 & 39.83 & 35.96 & 36.66 \\
\hline & 36.40 & 36.34 & 36.47 & 34.13 & 34.03 & 35.69 & 33.86 & 37.28 & 37.25 & 36.11 & 36.70 \\
\hline & 36.37 & 36.33 & 36.45 & 34.09 & 32.63 & 35.54 & 31.43 & 37.75 & 36.79 & 34.49 & 35.03 \\
\hline \multirow{3}{*}{ Nikon D600 ISO=3200 } & 34.18 & 34.70 & 34.79 & 33.62 & 31.78 & 35.57 & 33.46 & 34.55 & 35.50 & 33.94 & 33.72 \\
\hline & 35.07 & 36.20 & 36.37 & 34.48 & 35.16 & 36.70 & 36.09 & 35.99 & 37.24 & 34.33 & 34.70 \\
\hline & 37.13 & 39.33 & 39.49 & 35.41 & 39.98 & 39.28 & 39.86 & 38.62 & 41.18 & 38.87 & 37.98 \\
\hline \multirow{3}{*}{ Nikon D800 ISO=1600 } & 36.81 & 37.95 & 38.11 & 35.79 & 34.84 & 38.01 & 36.35 & 39.22 & 38.77 & 37.61 & 38.10 \\
\hline & 37.76 & 40.23 & 40.52 & 36.08 & 38.42 & 39.05 & 39.99 & 39.67 & 40.87 & 38.24 & 39.15 \\
\hline & 37.51 & 37.94 & 38.17 & 35.48 & 35.79 & 38.20 & 37.15 & 39.04 & 38.86 & 36.89 & 36.14 \\
\hline \multirow{3}{*}{ Nikon D800 ISO=3200 } & 35.05 & 37.55 & 37.69 & 34.08 & 38.36 & 38.07 & 38.60 & 38.28 & 39.94 & 37.20 & 36.93 \\
\hline & 34.07 & 35.91 & 35.90 & 33.70 & 35.53 & 35.72 & 36.04 & 37.18 & 36.78 & 35.67 & 35.80 \\
\hline & 34.42 & 38.15 & 38.21 & 33.31 & 40.05 & 36.76 & 39.73 & 38.85 & 39.78 & 38.09 & 37.49 \\
\hline \multirow{3}{*}{ Nikon D800 ISO=6400 } & 31.13 & 32.69 & 32.81 & 29.83 & 34.08 & 33.49 & 33.29 & 32.75 & 33.34 & 32.24 & 31.94 \\
\hline & 31.22 & 32.33 & 32.33 & 30.55 & 32.13 & 32.79 & 31.16 & 33.24 & 33.29 & 32.59 & 32.51 \\
\hline & 30.97 & 32.29 & 32.29 & 30.09 & 31.52 & 32.86 & 31.98 & 32.89 & 33.22 & 33.14 & 32.91 \\
\hline Average & 35.19 & 36.46 & 36.61 & 33.86 & 35.33 & 36.43 & 35.77 & 36.73 & 37.51 & 35.69 & 35.72 \\
\hline
\end{tabular}

Table 19: Different methods on the BSD68 for different noise levels (i.e., 15, 25 and 50).

\begin{tabular}{|c|c|c|c|}
\hline Methods & 15 & 25 & 50 \\
\hline DnCNN-B [258] & 31.61 & 29.16 & 26.23 \\
\hline FFDNet [260] & 31.62 & 29.19 & 26.30 \\
\hline SCNN [90] & 31.48 & 29.03 & 26.08 \\
\hline ADNet-B [206] & 31.56 & 29.14 & 26.24 \\
\hline DnCNN-SURE-T [191] & - & 29.00 & 25.95 \\
\hline DnCNN-MSE-GT [191] & - & 29.20 & 26.22 \\
\hline G2G1(LM,BSD) [27] & 31.55 & 28.93 & 25.73 \\
\hline
\end{tabular}


Table 20: Average PSNR (dB) results of different methods on Set12 with noise levels of 25 and 50.

\begin{tabular}{|c|c|c|c|c|c|c|c|c|c|c|c|c|c|}
\hline Images & C.man & House & Peppers & Starfish & Monarch & Airplane & Parrot & Lena & Barbara & Boat & Man & Couple & Average \\
\hline Noise Level & \multicolumn{13}{|c|}{$\sigma=25$} \\
\hline DnCNN-B 258 & 29.94 & 33.05 & 30.84 & 29.34 & 30.25 & 29.09 & 29.35 & 32.42 & 29.69 & 30.20 & 30.09 & 30.10 & 30.36 \\
\hline FFDNet [260 & 30.10 & 33.28 & 30.93 & 29.32 & 30.08 & 29.04 & 29.44 & 32.57 & 30.01 & 30.25 & 30.11 & 30.20 & 30.44 \\
\hline ADNet-B [206 & 29.94 & 33.38 & 30.99 & 29.22 & 30.38 & 29.16 & 29.41 & 32.59 & 30.05 & 30.28 & 30.01 & 30.15 & 30.46 \\
\hline DudeNet-B 208 & 30.01 & 33.15 & 30.87 & 29.39 & 30.31 & 29.07 & 29.40 & 32.42 & 29.76 & 30.18 & 30.03 & 30.06 & 30.39 \\
\hline DNCNN-SURE-T [191] & 29.86 & 32.73 & 30.57 & 29.11 & 30.13 & 28.93 & 29.26 & 32.08 & 29.44 & 29.86 & 29.91 & 29.78 & 30.14 \\
\hline DNCNN-MSE-GT [191] & 30.14 & 33.16 & 30.84 & 29.40 & 30.45 & 29.11 & 29.36 & 32.44 & 29.91 & 30.11 & 30.08 & 30.06 & 30.42 \\
\hline Noise Level & \multicolumn{13}{|c|}{$\sigma=50$} \\
\hline DnCNN-B [258] & 27.03 & 30.02 & 27.39 & 25.72 & 26.83 & 25.89 & 26.48 & 29.38 & 26.38 & 27.23 & 27.23 & 26.91 & 27.21 \\
\hline FFDNet [260 & 27.05 & 30.37 & 27.54 & 25.75 & 26.81 & 25.89 & 26.57 & 29.66 & 26.45 & 27.33 & 27.29 & 27.08 & 27.32 \\
\hline ADNet-B 206 & 27.22 & 30.43 & 27.70 & 25.63 & 26.92 & 26.03 & 26.56 & 29.53 & 26.51 & 27.22 & 27.19 & 27.05 & 27.33 \\
\hline DudeNet-B [208 & 27.19 & 30.11 & 27.50 & 25.69 & 26.82 & 25.85 & 26.46 & 29.35 & 26.38 & 27.20 & 27.13 & 26.90 & 27.22 \\
\hline DNCNN-SURE-T [191] & 26.47 & 29.20 & 26.78 & 25.39 & 26.53 & 25.65 & 26.21 & 28.81 & 25.23 & 26.79 & 26.97 & 26.48 & 26.71 \\
\hline DNCNN-MSE-GT [191] & 27.03 & 29.92 & 27.27 & 25.65 & 26.95 & 25.93 & 26.43 & 29.31 & 26.17 & 27.12 & 27.22 & 26.94 & 27.16 \\
\hline
\end{tabular}

Table 21: Different methods on the VggFace2and WebFace for image denoising.

\begin{tabular}{|c|c|c|c|c|}
\hline \multirow{2}{*}{ Methods } & \multicolumn{2}{|c|}{ VggFace2 [26 } & \multicolumn{2}{c|}{ WebFace [244 } \\
\cline { 2 - 5 } & $4 \times$ & $8 \times$ & $4 \times$ & $8 \times$ \\
\hline DnCNN [258 & 26.73 & 23.29 & 28.35 & 24.75 \\
\hline MemNet [197 & 26.85 & 23.31 & 28.57 & 24.77 \\
\hline WarpNet [127] & 28.55 & 24.10 & 32.31 & 27.21 \\
\hline
\end{tabular}

3) Combining local and global information can enhance the memory abilities of the shallow layers on deep layers to better filter the noise. Two methods for addressing this problem are residual operation and recursive operation.

4) Single processing methods can be used to suppress the noise. The single processing technique fused into the deep $\mathrm{CNN}$ can achieve excellent performance. For example, the wavelet technique is gathered into the U-Net to deal with image restoration [137].

5) Data augmentation, such as horizontal flip, vertical flip and color jittering, can help the denoising methods learn more types of noise, which can enhance the expressive ability of the denoising models. Additionally, using the GAN to construct virtual noisy images is also useful for image denoising.

6) Transfer learning, graph and neural architecture search methods can obtain good denoising results.

7) Improving the hardware or camera mechanism can reduce the effect of noise on the captured image.

Compressing deep neural networks has achieved great success in improving the efficiency of denoising. Reducing the depth or the width of deep neural networks can reduce the complexity of these networks in image denoising. Also, the use of small convolutional kernel and group convolution can reduce the number of parameters, thereby accelerating the speed of training. The fusion of dimension reduction methods, such as principal component analysis (PCA) and CNN, can also lead to improvements in denoising efficiency.

For resolving complex noisy images, step-by-step processing is a very popular method. For example, using a two-step mechanism is a way of dealing with a noisy image with low-resolution. The first step involves the recovery of a high-resolution image by a CNN. The second step uses a novel $\mathrm{CNN}$ to filter the noise of the high-resolution image. In the example above, the two CNNs 
are implemented via a cascade operation. This two-step mechanism is ideal for unsupervised noise tasks, such as real noisy images and blind denoising. That is, the first step relies on a CNN with optimization algorithms, i.e., maximum a posteriori, to estimate the noise as ground truth (referred as a label). The second step utilized another $\mathrm{CNN}$ and obtained ground truth to train a denoising model for real-noisy image denoising or blind denoising. The self-supervised learning fused into the $\mathrm{CNN}$ is a good choice for real-noisy image denoising or blind denoising.

Although deep learning techniques have attained great success in these three scenarios, there are still challenges in the field of image denoising. These include:

1) Deeper denoising networks require more memory resources.

2) Training deeper denoising networks is not a stable solution for real noisy image, unpaired noisy image and multi-degradation tasks.

3) Real noisy images are not easily captured, which results in inadequate training samples.

4) Deep CNNs are difficult to solve unsupervised denoising tasks.

5) More accurate metrics need to be found for image denoising. PSNR and SSIM are popular metrics for the task of image restoration. PSNR suffers from excessive smoothing, which is very difficult to recognize indistinguishable images. SSIM depends on brightness, contrast and structure, and therefore cannot accurately evaluate image perceptual quality.

\section{Conclusion}

In this paper, we compare, study and summarize the deep networks used for on image denoising. First, we show the basic frameworks of deep learning for image denoising. Then, we present the deep learning techniques for noisy tasks, including additive white noisy images, blind denoising, real noisy images and hybrid noisy images. Next, for each category of noisy tasks, we analyze the motivation and theory of denoising networks. Next, we compare the denoising results, efficiency and visual effects of different networks on benchmark datasets, and then perform a cross-comparison of the different types of image denoising methods with different types of noise. Finally, some potential areas for further research are suggested, and the challenges of deep learning in image denoising are discussed.

Over the past few years, Gaussian noisy image denoising techniques have achieved great success, particularly in scenarios where the Gaussian noise is regular. However, in the real world the noise is complex and irregular. Improving the hardware devices in order to better suppress the noise for capturing a high-quality image is very important. Moreover, the obtained image may be blurry, low-resolution and corrupted. Therefore, it is critical to determine how to effectively recover the latent clean image from the superposed noisy image. Furthermore, while the use of deep learning techniques to learn features requires the ground truth, the obtained real noisy images do not have the ground truth. These are urgent challenges that researches and scholars need to address.

\section{Acknowledgments}

This paper is partially supported by the National Natural Science Foundation of China under Grant No. 61876051, in part by Shenzhen Municipal Science and Technology Innovation, Council 
under Grant No. JSGG20190220153602271 and in part by the Natural Science Foundation of Guang dong Province under Grant No. 2019A1515011811.

\section{References}

\section{References}

[1] Abadi, M., Barham, P., Chen, J., Chen, Z., Davis, A., Dean, J., Devin, M., Ghemawat, S., Irving, G., Isard, M., et al., 2016. Tensorflow: A system for large-scale machine learning. In: 12th Symposium on Operating Systems Design and Implementation. pp. 265-283.

[2] Abbasi, A., Monadjemi, A., Fang, L., Rabbani, H., Zhang, Y., 2019. Three-dimensional optical coherence tomography image denoising through multi-input fully-convolutional networks. Computers in Biology and Medicine 108, 1-8.

[3] Abdelhamed, A., Lin, S., Brown, M. S., 2018. A high-quality denoising dataset for smartphone cameras. In: Proceedings of the IEEE Conference on Computer Vision and Pattern Recognition. pp. 1692-1700.

[4] Abiko, R., Ikehara, M., 2019. Blind denoising of mixed gaussian-impulse noise by single cnn. In: ICASSP 2019-2019 IEEE International Conference on Acoustics, Speech and Signal Processing (ICASSP). IEEE, pp. 1717-1721.

[5] ABSoft, N., 2017. Neat image.

[6] Aharon, M., Elad, M., Bruckstein, A., 2006. K-svd: An algorithm for designing overcomplete dictionaries for sparse representation. IEEE Transactions on Signal Processing 54 (11), 4311-4322.

[7] Ahn, B., Cho, N. I., 2017. Block-matching convolutional neural network for image denoising. arXiv preprint arXiv: 1704.00524.

[8] Ahn, B., Kim, Y., Park, G., Cho, N. I., 2018. Block-matching convolutional neural network (bmcnn): Improving cnn-based denoising by block-matched inputs. In: 2018 Asia-Pacific Signal and Information Processing Association Annual Summit and Conference (APSIPA ASC). IEEE, pp. 516-525.

[9] Aittala, M., Durand, F., 2018. Burst image deblurring using permutation invariant convolutional neural networks. In: Proceedings of the European Conference on Computer Vision (ECCV). pp. 731-747.

[10] Aljadaany, R., Pal, D. K., Savvides, M., 2019. Proximal splitting networks for image restoration. arXiv preprint arXiv:1903.07154.

[11] Anwar, S., Barnes, N., 2019. Real image denoising with feature attention. arXiv preprint arXiv:1904.07396.

[12] Anwar, S., Huynh, C. P., Porikli, F., 2017. Chaining identity mapping modules for image denoising. arXiv preprint arXiv:1712.02933.

[13] Bae, W., Yoo, J., Chul Ye, J., 2017. Beyond deep residual learning for image restoration: Persistent homologyguided manifold simplification. In: Proceedings of the IEEE Conference on Computer Vision and Pattern Recognition Workshops. pp. 145-153.

[14] Bako, S., Vogels, T., McWilliams, B., Meyer, M., Novák, J., Harvill, A., Sen, P., Derose, T., Rousselle, F., 2017. Kernel-predicting convolutional networks for denoising monte carlo renderings. ACM Transactions on Graphics (TOG) 36 (4), 97.

[15] Bedini, L., Tonazzini, A., 1990. Neural network use in maximum entropy image restoration. Image and Vision Computing 8 (2), 108-114.

[16] Bedini, L., Tonazzini, A., 1992. Image restoration preserving discontinuities: the bayesian approach and neural networks. Image and Vision Computing 10 (2), 108-118.

[17] Bengio, Y., Lamblin, P., Popovici, D., Larochelle, H., 2007. Greedy layer-wise training of deep networks. In: Advances in Neural Information Processing Systems. pp. 153-160.

[18] Bergstra, J., Breuleux, O., Bastien, F., Lamblin, P., Pascanu, R., Desjardins, G., Turian, J., Warde-Farley, D., Bengio, Y., 2010. Theano: a cpu and gpu math expression compiler. In: Proceedings of the Python for Scientific Computing Conference (SciPy). Vol. 4. Austin, TX.

[19] Bernstein, R., 1987. Adaptive nonlinear filters for simultaneous removal of different kinds of noise in images. IEEE Transactions on Circuits and Systems 34 (11), 1275-1291. 
[20] Bigdeli, S. A., Zwicker, M., 2017. Image restoration using autoencoding priors. arXiv preprint arXiv:1703.09964.

[21] Bigdeli, S. A., Zwicker, M., Favaro, P., Jin, M., 2017. Deep mean-shift priors for image restoration. In: Advances in Neural Information Processing Systems. pp. 763-772.

[22] Bottou, L., 2010. Large-scale machine learning with stochastic gradient descent. In: Proceedings of COMPSTAT'2010. Springer, pp. 177-186.

[23] Broaddus, C., Krull, A., Weigert, M., Schmidt, U., Myers, G., 2020. Removing structured noise with selfsupervised blind-spot networks. In: 2020 IEEE 17th International Symposium on Biomedical Imaging (ISBI). IEEE, pp. 159-163.

[24] Brooks, T., Mildenhall, B., Xue, T., Chen, J., Sharlet, D., Barron, J. T., 2019. Unprocessing images for learned raw denoising. In: Proceedings of the IEEE Conference on Computer Vision and Pattern Recognition. pp. 11036-11045.

[25] Burger, H. C., Schuler, C. J., Harmeling, S., 2012. Image denoising: Can plain neural networks compete with bm3d? In: 2012 IEEE Conference on Computer Vision and Pattern Recognition. IEEE, pp. 2392-2399.

[26] Cao, Q., Shen, L., Xie, W., Parkhi, O. M., Zisserman, A., 2018. Vggface2: A dataset for recognising faces across pose and age. In: 2018 13th IEEE International Conference on Automatic Face \& Gesture Recognition (FG 2018). IEEE, pp. 67-74.

[27] Cha, S., Park, T., Moon, T., 2019. Gan2gan: Generative noise learning for blind image denoising with single noisy images. arXiv preprint arXiv:1905.10488.

[28] Chang, Y., Yan, L., Fang, H., Zhong, S., Liao, W., 2018. Hsi-denet: Hyperspectral image restoration via convolutional neural network. IEEE Transactions on Geoscience and Remote Sensing 57 (2), 667-682.

[29] Chen, C., Xiong, Z., Tian, X., Wu, F., 2018. Deep boosting for image denoising. In: Proceedings of the European Conference on Computer Vision (ECCV). pp. 3-18.

[30] Chen, C., Xu, Z., 2018. Aerial-image denoising based on convolutional neural network with multi-scale residual learning approach. Information 9 (7), 169.

[31] Chen, J., Chen, J., Chao, H., Yang, M., 2018. Image blind denoising with generative adversarial network based noise modeling. In: Proceedings of the IEEE Conference on Computer Vision and Pattern Recognition. pp. 3155-3164.

[32] Chen, J., Hou, J., Chau, L.-P., 2018. Light field denoising via anisotropic parallax analysis in a cnn framework. IEEE Signal Processing Letters 25 (9), 1403-1407.

[33] Chen, X., Song, L., Yang, X., 2016. Deep rnns for video denoising. In: Applications of Digital Image Processing XXXIX. Vol. 9971. International Society for Optics and Photonics, p. 99711T.

[34] Chen, X., Zhan, S., Ji, D., Xu, L., Wu, C., Li, X., 2018. Image denoising via deep network based on edge enhancement. Journal of Ambient Intelligence and Humanized Computing, 1-11.

[35] Chen, Y., Pock, T., 2016. Trainable nonlinear reaction diffusion: A flexible framework for fast and effective image restoration. IEEE Transactions on Pattern Analysis and Machine Intelligence 39 (6), 1256-1272.

[36] Chen, Y., Yu, M., Jiang, G., Peng, Z., Chen, F., 2019. End-to-end single image enhancement based on a dual network cascade model. Journal of Visual Communication and Image Representation 61, 284-295.

[37] Chetlur, S., Woolley, C., Vandermersch, P., Cohen, J., Tran, J., Catanzaro, B., Shelhamer, E., 2014. cudnn: Efficient primitives for deep learning. arXiv preprint arXiv:1410.0759.

[38] Chiang, Y.-W., Sullivan, B., 1989. Multi-frame image restoration using a neural network. In: Proceedings of the 32nd Midwest Symposium on Circuits and Systems,. IEEE, pp. 744-747.

[39] Cho, S. I., Kang, S.-J., 2018. Gradient prior-aided cnn denoiser with separable convolution-based optimization of feature dimension. IEEE Transactions on Multimedia 21 (2), 484-493.

[40] Choi, K., Vania, M., Kim, S., 2019. Semi-supervised learning for low-dose ct image restoration with hierarchical deep generative adversarial network (hd-gan). In: 2019 41st Annual International Conference of the IEEE Engineering in Medicine and Biology Society (EMBC). IEEE, pp. 2683-2686.

[41] Chollet, F., et al., 2015. Keras.

[42] Couturier, R., Perrot, G., Salomon, M., 2018. Image denoising using a deep encoder-decoder network with skip connections. In: International Conference on Neural Information Processing. Springer, pp. 554-565.

[43] Cruz, C., Foi, A., Katkovnik, V., Egiazarian, K., 2018. Nonlocality-reinforced convolutional neural networks 
for image denoising. IEEE Signal Processing Letters 25 (8), 1216-1220.

[44] Cui, J., Gong, K., Guo, N., Wu, C., Meng, X., Kim, K., Zheng, K., Wu, Z., Fu, L., Xu, B., et al., 2019. Pet image denoising using unsupervised deep learning. European journal of nuclear medicine and molecular imaging 46 (13), 2780-2789.

[45] Dabov, K., Foi, A., Katkovnik, V., Egiazarian, K., 2007. Image denoising by sparse 3-d transform-domain collaborative filtering. IEEE Transactions on Image Processing 16 (8), 2080-2095.

[46] Davy, A., Ehret, T., Morel, J.-M., Arias, P., Facciolo, G., 2018. Non-local video denoising by cnn. arXiv preprint arXiv:1811.12758.

[47] de Figueiredo, M. T., Leitao, J. M., 1992. Image restoration using neural networks. In: [Proceedings] ICASSP92: 1992 IEEE International Conference on Acoustics, Speech, and Signal Processing. Vol. 2. IEEE, pp. 409412.

[48] de Ridder, D., Duin, R. P., Verbeek, P. W., Van Vliet, L., 1999. The applicability of neural networks to nonlinear image processing. Pattern Analysis \& Applications 2 (2), 111-128.

[49] Dong, W., Zhang, L., Shi, G., Li, X., 2012. Nonlocally centralized sparse representation for image restoration. IEEE Transactions on Image Processing 22 (4), 1620-1630.

[50] Du, B., Wei, Q., Liu, R., 2019. An improved quantum-behaved particle swarm optimization for endmember extraction. IEEE Transactions on Geoscience and Remote Sensing.

[51] Du, H., Dong, L., Liu, M., Zhao, Y., Jia, W., Liu, X., Hui, M., Kong, L., Hao, Q., 2018. Image restoration based on deep convolutional network in wavefront coding imaging system. In: 2018 Digital Image Computing: Techniques and Applications (DICTA). IEEE, pp. 1-8.

[52] Duan, C., Cui, L., Chen, X., Wei, F., Zhu, C., Zhao, T., 2018. Attention-fused deep matching network for natural language inference. In: IJCAI. pp. 4033-4040.

[53] Ehret, T., Davy, A., Morel, J.-M., Facciolo, G., Arias, P., 2019. Model-blind video denoising via frame-toframe training. In: Proceedings of the IEEE Conference on Computer Vision and Pattern Recognition. pp. 11369-11378.

[54] Elad, M., Aharon, M., 2006. Image denoising via sparse and redundant representations over learned dictionaries. IEEE Transactions on Image Processing 15 (12), 3736-3745.

[55] Fan, E., 2000. Extended tanh-function method and its applications to nonlinear equations. Physics Letters A 277 (4-5), 212-218.

[56] Farooque, M. A., Rohankar, J. S., 2013. Survey on various noises and techniques for denoising the color image. International Journal of Application or Innovation in Engineering \& Management (IJAIEM) 2 (11), $217-221$.

[57] Franzen, R., 1999. Kodak lossless true color image suite. source: http://r0k. us/graphics/kodak 4.

[58] Fu, B., Zhao, X., Li, Y., Wang, X., Ren, Y., 2019. A convolutional neural networks denoising approach for salt and pepper noise. Multimedia Tools and Applications 78 (21), 30707-30721.

[59] Fukushima, K., 1980. Neocognitron: A self-organizing neural network model for a mechanism of pattern recognition unaffected by shift in position. Biological Cybernetics 36 (4), 193-202.

[60] Fukushima, K., Miyake, S., 1982. Neocognitron: A self-organizing neural network model for a mechanism of visual pattern recognition. In: Competition and Cooperation in Neural Nets. Springer, pp. 267-285.

[61] Gardner, E., Wallace, D., Stroud, N., 1989. Training with noise and the storage of correlated patterns in a neural network model. Journal of Physics A: Mathematical and General 22 (12), 2019.

[62] Gashi, D., Pereira, M., Vterkovska, V., 2017. Multi-scale context aggregation by dilated convolutions machine learning-project.

[63] Gholizadeh-Ansari, M., Alirezaie, J., Babyn, P., 2018. Low-dose ct denoising with dilated residual network. In: 2018 40th Annual International Conference of the IEEE Engineering in Medicine and Biology Society (EMBC). IEEE, pp. 5117-5120.

[64] Godard, C., Matzen, K., Uyttendaele, M., 2018. Deep burst denoising. In: Proceedings of the European Conference on Computer Vision (ECCV). pp. 538-554.

[65] Gondara, L., Wang, K., 2017. Recovering loss to followup information using denoising autoencoders. In: 2017 IEEE International Conference on Big Data (Big Data). IEEE, pp. 1936-1945.

[66] Gong, D., Zhang, Z., Shi, Q., Hengel, A. v. d., Shen, C., Zhang, Y., 2018. Learning an optimizer for image deconvolution. arXiv preprint arXiv:1804.03368. 
[67] Green, M., Marom, E. M., Konen, E., Kiryati, N., Mayer, A., 2018. Learning real noise for ultra-low dose lung ct denoising. In: International Workshop on Patch-based Techniques in Medical Imaging. Springer, pp. 3-11.

[68] Greenhill, D., Davies, E., 1994. Relative effectiveness of neural networks for image noise suppression. In: Machine Intelligence and Pattern Recognition. Vol. 16. Elsevier, pp. 367-378.

[69] Gu, S., Zhang, L., Zuo, W., Feng, X., 2014. Weighted nuclear norm minimization with application to image denoising. In: Proceedings of the IEEE Conference on Computer Vision and Pattern Recognition. pp. 28622869.

[70] Guan, J., Lai, R., Xiong, A., 2019. Wavelet deep neural network for stripe noise removal. IEEE Access 7 , 44544-44554.

[71] Guo, S., Yan, Z., Zhang, K., Zuo, W., Zhang, L., 2019. Toward convolutional blind denoising of real photographs. In: Proceedings of the IEEE Conference on Computer Vision and Pattern Recognition. pp. 17121722 .

[72] Guo, Z., Sun, Y., Jian, M., Zhang, X., 2018. Deep residual network with sparse feedback for image restoration. Applied Sciences 8 (12), 2417.

[73] Han, Y., Ye, J. C., 2018. Framing u-net via deep convolutional framelets: Application to sparse-view ct. IEEE Transactions on Medical Imaging 37 (6), 1418-1429.

[74] He, J., Dong, C., Qiao, Y., 2019. Multi-dimension modulation for image restoration with dynamic controllable residual learning. arXiv preprint arXiv:1912.05293.

[75] He, K., Zhang, X., Ren, S., Sun, J., 2016. Deep residual learning for image recognition. In: Proceedings of the IEEE Conference on Computer Vision and Pattern Recognition. pp. 770-778.

[76] Heckel, R., Huang, W., Hand, P., Voroninski, V., 2018. Rate-optimal denoising with deep neural networks. arXiv preprint arXiv:1805.08855.

[77] Heinrich, M. P., Stille, M., Buzug, T. M., 2018. Residual u-net convolutional neural network architecture for low-dose ct denoising. Current Directions in Biomedical Engineering 4 (1), 297-300.

[78] Hendriksen, A. A., Pelt, D. M., Batenburg, K. J., 2020. Noise2inverse: Self-supervised deep convolutional denoising for linear inverse problems in imaging. arXiv preprint arXiv:2001.11801.

[79] Hinton, G., Osindero, S., ???? The, y. 2006. a fast learning algorithm for deep belief nets. Neural Computation 18 (7).

[80] Hinton, G. E., Salakhutdinov, R. R., 2006. Reducing the dimensionality of data with neural networks. Science 313 (5786), 504-507.

[81] Hirose, Y., Yamashita, K., Hijiya, S., 1991. Back-propagation algorithm which varies the number of hidden units. Neural Networks 4 (1), 61-66.

[82] Hong, S.-W., Bao, P., 2000. An edge-preserving subband coding model based on non-adaptive and adaptive regularization. Image and Vision Computing 18 (8), 573-582.

[83] Hongqiang, M., Shiping, M., Yuelei, X., Mingming, Z., 2018. An adaptive image denoising method based on deep rectified denoising auto-encoder. In: Journal of Physics: Conference Series. Vol. 1060. IOP Publishing, p. 012048.

[84] Hore, A., Ziou, D., 2010. Image quality metrics: Psnr vs. ssim. In: 2010 20th International Conference on Pattern Recognition. IEEE, pp. 2366-2369.

[85] Hsu, C.-C., Lin, C.-W., 2017. Cnn-based joint clustering and representation learning with feature drift compensation for large-scale image data. IEEE Transactions on Multimedia 20 (2), 421-429.

[86] Hu, G., Yang, Y., Yi, D., Kittler, J., Christmas, W., Li, S. Z., Hospedales, T., 2015. When face recognition meets with deep learning: an evaluation of convolutional neural networks for face recognition. In: Proceedings of the IEEE international Conference on Computer Vision Workshops. pp. 142-150.

[87] HUANG, T., 1971. Stability of two-dimensional recursive filters(mathematical model for stability problem in two-dimensional recursive filtering).

[88] Ioffe, S., 2017. Batch renormalization: Towards reducing minibatch dependence in batch-normalized models. In: Advances In Neural Information Processing Systems. pp. 1945-1953.

[89] Ioffe, S., Szegedy, C., 2015. Batch normalization: Accelerating deep network training by reducing internal covariate shift. arXiv preprint arXiv:1502.03167.

[90] Isogawa, K., Ida, T., Shiodera, T., Takeguchi, T., 2017. Deep shrinkage convolutional neural network for 
adaptive noise reduction. IEEE Signal Processing Letters 25 (2), 224-228.

[91] Jaroensri, R., Biscarrat, C., Aittala, M., Durand, F., 2019. Generating training data for denoising real rgb images via camera pipeline simulation. arXiv preprint arXiv:1904.08825.

[92] Jarrett, K., Kavukcuoglu, K., Ranzato, M., LeCun, Y., 2009. What is the best multi-stage architecture for object recognition? In: 2009 IEEE 12th International Conference on Computer Vision. IEEE, pp. 2146-2153.

[93] Jeon, W., Jeong, W., Son, K., Yang, H., 2018. Speckle noise reduction for digital holographic images using multi-scale convolutional neural networks. Optics Letters 43 (17), 4240-4243.

[94] Jia, X., Chai, H., Guo, Y., Huang, Y., Zhao, B., 2018. Multiscale parallel feature extraction convolution neural network for image denoising. Journal of Electronic Imaging 27 (6), 063031.

[95] Jia, X., Liu, S., Feng, X., Zhang, L., 2019. Focnet: A fractional optimal control network for image denoising. In: Proceedings of the IEEE Conference on Computer Vision and Pattern Recognition. pp. 6054-6063.

[96] Jia, Y., Shelhamer, E., Donahue, J., Karayev, S., Long, J., Girshick, R., Guadarrama, S., Darrell, T., 2014. Caffe: Convolutional architecture for fast feature embedding. In: Proceedings of the 22nd ACM International Conference on Multimedia. ACM, pp. 675-678.

[97] Jian, W., Zhao, H., Bai, Z., Fan, X., 2018. Low-light remote sensing images enhancement algorithm based on fully convolutional neural network. In: China High Resolution Earth Observation Conference. Springer, pp. $56-65$.

[98] Jiang, D., Dou, W., Vosters, L., Xu, X., Sun, Y., Tan, T., 2018. Denoising of 3d magnetic resonance images with multi-channel residual learning of convolutional neural network. Japanese Journal of Radiology 36 (9), 566-574.

[99] Jiang, L., Jing, Y., Hu, S., Ge, B., Xiao, W., 2018. Deep refinement network for natural low-light image enhancement in symmetric pathways. Symmetry 10 (10), 491.

[100] Jiao, J., Tu, W.-C., He, S., Lau, R. W., 2017. Formresnet: Formatted residual learning for image restoration. In: Proceedings of the IEEE Conference on Computer Vision and Pattern Recognition Workshops. pp. 38-46.

[101] Jifara, W., Jiang, F., Rho, S., Cheng, M., Liu, S., 2019. Medical image denoising using convolutional neural network: a residual learning approach. The Journal of Supercomputing 75 (2), 704-718.

[102] Jin, K. H., McCann, M. T., Froustey, E., Unser, M., 2017. Deep convolutional neural network for inverse problems in imaging. IEEE Transactions on Image Processing 26 (9), 4509-4522.

[103] Kadimesetty, V. S., Gutta, S., Ganapathy, S., Yalavarthy, P. K., 2018. Convolutional neural network-based robust denoising of low-dose computed tomography perfusion maps. IEEE Transactions on Radiation and Plasma Medical Sciences 3 (2), 137-152.

[104] Karlik, B., Olgac, A. V., 2011. Performance analysis of various activation functions in generalized mlp architectures of neural networks. International Journal of Artificial Intelligence and Expert Systems 1 (4), 111-122.

[105] Khan, S., Khan, K. S., Shin, S. Y., 2019. Symbol denoising in high order m-qam using residual learning of deep cnn. In: 2019 16th IEEE Annual Consumer Communications \& Networking Conference (CCNC). IEEE, pp. 1-6.

[106] Khaw, H. Y., Soon, F. C., Chuah, J. H., Chow, C.-O., 2017. Image noise types recognition using convolutional neural network with principal components analysis. IET Image Processing 11 (12), 1238-1245.

[107] Khoroushadi, M., Sadegh, M., 2018. Enhancement in low-dose computed tomography through image denoising techniques: Wavelets and deep learning. Ph.D. thesis, ProQuest Dissertations Publishing.

[108] Kokkinos, F., Lefkimmiatis, S., 2019. Iterative joint image demosaicking and denoising using a residual denoising network. IEEE Transactions on Image Processing.

[109] Krizhevsky, A., Sutskever, I., Hinton, G. E., 2012. Imagenet classification with deep convolutional neural networks. In: Advances in Neural Information Processing Systems. pp. 1097-1105.

[110] Kutzner, C., Páll, S., Fechner, M., Esztermann, A., de Groot, B. L., Grubmüller, H., 2019. More bang for your buck: improved use of gpu nodes for gromacs 2018. arXiv preprint arXiv:1903.05918.

[111] Latif, G., Iskandar, D. A., Alghazo, J., Butt, M., Khan, A. H., 2018. Deep cnn based mr image denoising for tumor segmentation using watershed transform. International Journal of Engineering \& Technology 7 (2.3), $37-42$.

[112] Lebrun, M., Colom, M., Morel, J.-M., 2015. The noise clinic: a blind image denoising algorithm. Image Processing On Line 5, 1-54. 
[113] LeCun, Y., Bottou, L., Bengio, Y., Haffner, P., et al., 1998. Gradient-based learning applied to document recognition. Proceedings of the IEEE 86 (11), 2278-2324.

[114] Lee, C.-C., de Gyvez, J. P., 1996. Color image processing in a cellular neural-network environment. IEEE Transactions on Neural Networks 7 (5), 1086-1098.

[115] Lee, D., Yun, S., Choi, S., Yoo, H., Yang, M.-H., Oh, S., 2018. Unsupervised holistic image generation from key local patches. In: Proceedings of the European Conference on Computer Vision (ECCV). pp. 19-35.

[116] Lefkimmiatis, S., 2017. Non-local color image denoising with convolutional neural networks. In: Proceedings of the IEEE Conference on Computer Vision and Pattern Recognition. pp. 3587-3596.

[117] Lei, Y., Yuan, W., Wang, H., Wenhu, Y., Bo, W., 2016. A skin segmentation algorithm based on stacked autoencoders. IEEE Transactions on Multimedia 19 (4), 740-749.

[118] Li, H., Yang, W., Yong, X., 2018. Deep learning for ground-roll noise attenuation. In: SEG Technical Program Expanded Abstracts 2018. Society of Exploration Geophysicists, pp. 1981-1985.

[119] Li, J., Li, M., Lu, G., Zhang, B., Yin, H., Zhang, D., 2020. Similarity and diversity induced paired projection for cross-modal retrieval. Information Sciences.

[120] Li, J., Lu, G., Zhang, B., You, J., Zhang, D., 2019. Shared linear encoder-based multikernel gaussian process latent variable model for visual classification. IEEE transactions on cybernetics.

[121] Li, J., Zhang, B., Zhang, D., 2017. Shared autoencoder gaussian process latent variable model for visual classification. IEEE transactions on neural networks and learning systems 29 (9), 4272-4286.

[122] Li, L., Wu, J., Jin, X., 2018. Cnn denoising for medical image based on wavelet domain. In: 2018 9th International Conference on Information Technology in Medicine and Education (ITME). IEEE, pp. 105-109.

[123] Li, M., Hsu, W., Xie, X., Cong, J., Gao, W., 2020. Sacnn: Self-attention convolutional neural network for low-dose ct denoising with self-supervised perceptual loss network. IEEE Transactions on Medical Imaging.

[124] Li, Q., Cai, W., Wang, X., Zhou, Y., Feng, D. D., Chen, M., 2014. Medical image classification with convolutional neural network. In: 2014 13th International Conference on Control Automation Robotics \& Vision (ICARCV). IEEE, pp. 844-848.

[125] Li, S., He, F., Du, B., Zhang, L., Xu, Y., Tao, D., 2019. Fast spatio-temporal residual network for video super-resolution. arXiv preprint arXiv:1904.02870.

[126] Li, X., Du, B., Xu, C., Zhang, Y., Zhang, L., Tao, D., 2020. Robust learning with imperfect privileged information. Artificial Intelligence 282, 103246.

[127] Li, X., Liu, M., Ye, Y., Zuo, W., Lin, L., Yang, R., 2018. Learning warped guidance for blind face restoration. In: Proceedings of the European Conference on Computer Vision (ECCV). pp. 272-289.

[128] Li, Z., Wu, J., 2019. Learning deep cnn denoiser priors for depth image inpainting. Applied Sciences 9 (6), 1103.

[129] Liang, J., Liu, R., 2015. Stacked denoising autoencoder and dropout together to prevent overfitting in deep neural network. In: 2015 8th International Congress on Image and Signal Processing (CISP). IEEE, pp. 697701.

[130] Liang, X., Zhang, D., Lu, G., Guo, Z., Luo, N., 2019. A novel multicamera system for high-speed touchless palm recognition. IEEE Transactions on Systems, Man, and Cybernetics: Systems.

[131] Lin, K., Li, T. H., Liu, S., Li, G., 2019. Real photographs denoising with noise domain adaptation and attentive generative adversarial network. In: Proceedings of the IEEE Conference on Computer Vision and Pattern Recognition Workshops. pp. 0-0.

[132] Lin, M., Chen, Q., Yan, S., 2013. Network in network. arXiv preprint arXiv:1312.4400.

[133] Litjens, G., Kooi, T., Bejnordi, B. E., Setio, A. A. A., Ciompi, F., Ghafoorian, M., Van Der Laak, J. A., Van Ginneken, B., Sánchez, C. I., 2017. A survey on deep learning in medical image analysis. Medical Image Analysis 42, 60-88.

[134] Liu, D., Wen, B., Liu, X., Wang, Z., Huang, T. S., 2017. When image denoising meets high-level vision tasks: A deep learning approach. arXiv preprint arXiv:1706.04284.

[135] Liu, P., Fang, R., 2017. Wide inference network for image denoising via learning pixel-distribution prior. arXiv preprint arXiv:1707.05414.

[136] Liu, P., Li, Y., El Basha, M. D., Fang, R., 2018. Neural network evolution using expedited genetic algorithm for medical image denoising. In: International Conference on Medical Image Computing and Computer-Assisted 
Intervention. Springer, pp. 12-20.

[137] Liu, P., Zhang, H., Zhang, K., Lin, L., Zuo, W., 2018. Multi-level wavelet-cnn for image restoration. In: Proceedings of the IEEE Conference on Computer Vision and Pattern Recognition Workshops. pp. 773-782.

[138] Liu, Q., Lu, X., He, Z., Zhang, C., Chen, W.-S., 2017. Deep convolutional neural networks for thermal infrared object tracking. Knowledge-Based Systems 134, 189-198.

[139] Liu, W., Lee, J., 2019. A 3-d atrous convolution neural network for hyperspectral image denoising. IEEE Transactions on Geoscience and Remote Sensing.

[140] Liu, X., Suganuma, M., Sun, Z., Okatani, T., 2019. Dual residual networks leveraging the potential of paired operations for image restoration. In: Proceedings of the IEEE Conference on Computer Vision and Pattern Recognition. pp. 7007-7016.

[141] Lo, S.-C., Lou, S.-L., Lin, J.-S., Freedman, M. T., Chien, M. V., Mun, S. K., 1995. Artificial convolution neural network techniques and applications for lung nodule detection. IEEE Transactions on Medical Imaging 14 (4), 711-718.

[142] LOO TIANG KUAN, L., 2017. Survey of deep neural networks in blind denoising using different architectures and different labels. Ph.D. thesis.

[143] Lu, Y., Lai, Z., Li, X., Wong, W. K., Yuan, C., Zhang, D., 2018. Low-rank 2-d neighborhood preserving projection for enhanced robust image representation. IEEE Transactions on Cybernetics 49 (5), 1859-1872.

[144] Lu, Y., Wong, W., Lai, Z., Li, X., 2019. Robust flexible preserving embedding. IEEE Transactions on Cybernetics.

[145] Lu, Z., Yu, Z., Ya-Li, P., Shi-Gang, L., Xiaojun, W., Gang, L., Yuan, R., 2018. Fast single image superresolution via dilated residual networks. IEEE Access.

[146] Lucas, A., Iliadis, M., Molina, R., Katsaggelos, A. K., 2018. Using deep neural networks for inverse problems in imaging: beyond analytical methods. IEEE Signal Processing Magazine 35 (1), 20-36.

[147] Ma, K., Duanmu, Z., Wu, Q., Wang, Z., Yong, H., Li, H., Zhang, L., 2016. Waterloo exploration database: New challenges for image quality assessment models. IEEE Transactions on Image Processing 26 (2), 1004-1016.

[148] Ma, Y., Chen, X., Zhu, W., Cheng, X., Xiang, D., Shi, F., 2018. Speckle noise reduction in optical coherence tomography images based on edge-sensitive cgan. Biomedical Optics Express 9 (11), 5129-5146.

[149] Mafi, M., Martin, H., Cabrerizo, M., Andrian, J., Barreto, A., Adjouadi, M., 2018. A comprehensive survey on impulse and gaussian denoising filters for digital images. Signal Processing.

[150] Mairal, J., Bach, F. R., Ponce, J., Sapiro, G., Zisserman, A., 2009. Non-local sparse models for image restoration. In: ICCV. Vol. 29. Citeseer, pp. 54-62.

[151] Majumdar, A., 2018. Blind denoising autoencoder. IEEE Transactions on Neural Networks and Learning Systems 30 (1), 312-317.

[152] Mao, X., Shen, C., Yang, Y.-B., 2016. Image restoration using very deep convolutional encoder-decoder networks with symmetric skip connections. In: Advances in Neural Information Processing Systems. pp. 28022810.

[153] Marinč, T., Srinivasan, V., Gül, S., Hellge, C., Samek, W., 2019. Multi-kernel prediction networks for denoising of burst images. In: 2019 IEEE International Conference on Image Processing (ICIP). IEEE, pp. 2404-2408.

[154] Marreiros, A. C., Daunizeau, J., Kiebel, S. J., Friston, K. J., 2008. Population dynamics: variance and the sigmoid activation function. Neuroimage 42 (1), 147-157.

[155] McCann, M. T., Jin, K. H., Unser, M., 2017. Convolutional neural networks for inverse problems in imaging: A review. IEEE Signal Processing Magazine 34 (6), 85-95.

[156] Meinhardt, T., Moller, M., Hazirbas, C., Cremers, D., 2017. Learning proximal operators: Using denoising networks for regularizing inverse imaging problems. In: Proceedings of the IEEE International Conference on Computer Vision. pp. 1781-1790.

[157] Meng, M., Li, S., Yao, L., Li, D., Zhu, M., Gao, Q., Xie, Q., Zhao, Q., Bian, Z., Huang, J., et al., 2020. Semisupervised learned sinogram restoration network for low-dose ct image reconstruction. In: Medical Imaging 2020: Physics of Medical Imaging. Vol. 11312. International Society for Optics and Photonics, p. 113120B.

[158] Mildenhall, B., Barron, J. T., Chen, J., Sharlet, D., Ng, R., Carroll, R., 2018. Burst denoising with kernel prediction networks. In: Proceedings of the IEEE Conference on Computer Vision and Pattern Recognition. pp. 2502-2510. 
[159] Nair, V., Hinton, G. E., 2010. Rectified linear units improve restricted boltzmann machines. In: Proceedings of the 27th international conference on machine learning (ICML-10). pp. 807-814.

[160] Nam, S., Hwang, Y., Matsushita, Y., Joo Kim, S., 2016. A holistic approach to cross-channel image noise modeling and its application to image denoising. In: Proceedings of the IEEE Conference on Computer Vision and Pattern Recognition. pp. 1683-1691.

[161] Nossek, J., Roska, T., 1993. Special issue on cellular neural networks-introduction.

[162] Nvidia, C., 2011. Nvidia cuda c programming guide. Nvidia Corporation 120 (18), 8.

[163] Osher, S., Burger, M., Goldfarb, D., Xu, J., Yin, W., 2005. An iterative regularization method for total variationbased image restoration. Multiscale Modeling \& Simulation 4 (2), 460-489.

[164] Paik, J. K., Katsaggelos, A. K., 1992. Image restoration using a modified hopfield network. IEEE Transactions on Image Processing 1 (1), 49-63.

[165] Panda, A., Naskar, R., Pal, S., 2018. Exponential linear unit dilated residual network for digital image denoising. Journal of Electronic Imaging 27 (5), 053024.

[166] Pardasani, R., Shreemali, U., 2018. Image denoising and super-resolution using residual learning of deep convolutional network. arXiv preprint arXiv:1809.08229.

[167] Park, J. H., Kim, J. H., Cho, S. I., 2018. The analysis of cnn structure for image denoising. In: 2018 International SoC Design Conference (ISOCC). IEEE, pp. 220-221.

[168] Paszke, A., Gross, S., Chintala, S., Chanan, G., Yang, E., DeVito, Z., Lin, Z., Desmaison, A., Antiga, L., Lerer, A., 2017. Automatic differentiation in pytorch.

[169] Peng, Y., Zhang, L., Liu, S., Wu, X., Zhang, Y., Wang, X., 2019. Dilated residual networks with symmetric skip connection for image denoising. Neurocomputing 345, 67-76.

[170] Pitas, I., Venetsanopoulos, A., 1986. Nonlinear mean filters in image processing. IEEE Transactions on Acoustics, Speech, and Signal Processing 34 (3), 573-584.

[171] Plotz, T., Roth, S., 2017. Benchmarking denoising algorithms with real photographs. In: Proceedings of the IEEE Conference on Computer Vision and Pattern Recognition. pp. 1586-1595.

[172] Priyanka, S. A., Wang, Y.-K., 2019. Fully symmetric convolutional network for effective image denoising. Applied Sciences 9 (4), 778.

[173] Radford, A., Metz, L., Chintala, S., 2015. Unsupervised representation learning with deep convolutional generative adversarial networks. arXiv preprint arXiv:1511.06434.

[174] Ran, M., Hu, J., Chen, Y., Chen, H., Sun, H., Zhou, J., Zhang, Y., 2019. Denoising of 3d magnetic resonance images using a residual encoder-decoder wasserstein generative adversarial network. Medical Image Analysis $55,165-180$.

[175] Remez, T., Litany, O., Giryes, R., Bronstein, A. M., 2018. Class-aware fully convolutional gaussian and poisson denoising. IEEE Transactions on Image Processing 27 (11), 5707-5722.

[176] Ren, D., Shang, W., Zhu, P., Hu, Q., Meng, D., Zuo, W., 2020. Single image deraining using bilateral recurrent network. IEEE Transactions on Image Processing.

[177] Ren, D., Zuo, W., Hu, Q., Zhu, P., Meng, D., 2019. Progressive image deraining networks: a better and simpler baseline. In: Proceedings of the IEEE Conference on Computer Vision and Pattern Recognition. pp. 39373946.

[178] Ren, D., Zuo, W., Zhang, D., Zhang, L., Yang, M.-H., 2019. Simultaneous fidelity and regularization learning for image restoration. IEEE Transactions on Pattern Analysis and Machine Intelligence.

[179] Ren, W., Liu, S., Ma, L., Xu, Q., Xu, X., Cao, X., Du, J., Yang, M.-H., 2019. Low-light image enhancement via a deep hybrid network. IEEE Transactions on Image Processing 28 (9), 4364-4375.

[180] Ren, W., Pan, J., Zhang, H., Cao, X., Yang, M.-H., 2020. Single image dehazing via multi-scale convolutional neural networks with holistic edges. International Journal of Computer Vision 128 (1), 240-259.

[181] Roth, S., Black, M. J., 2005. Fields of experts: A framework for learning image priors. In: 2005 IEEE Computer Society Conference on Computer Vision and Pattern Recognition (CVPR'05). Vol. 2. Citeseer, pp. 860-867.

[182] Sadda, P., Qarni, T., 2018. Real-time medical video denoising with deep learning: application to angiography. International journal of applied information systems 12 (13), 22.

[183] Schmidhuber, J., 2015. Deep learning in neural networks: An overview. Neural Networks 61, 85-117.

[184] Schmidt, U., Roth, S., 2014. Shrinkage fields for effective image restoration. In: Proceedings of the IEEE 
Conference on Computer Vision and Pattern Recognition. pp. 2774-2781.

[185] Shantia, A., Timmers, R., Schomaker, L., Wiering, M., 2015. Indoor localization by denoising autoencoders and semi-supervised learning in 3d simulated environment. In: 2015 International Joint Conference on Neural Networks (IJCNN). IEEE, pp. 1-7.

[186] Sheremet, O., Sheremet, K., Sadovoi, O., Sokhina, Y., 2018. Convolutional neural networks for image denoising in infocommunication systems. In: 2018 International Scientific-Practical Conference Problems of Infocommunications. Science and Technology (PIC S\&T). IEEE, pp. 429-432.

[187] Shwartz-Ziv, R., Tishby, N., 2017. Opening the black box of deep neural networks via information. arXiv preprint arXiv:1703.00810.

[188] Si, X., Yuan, Y., 2018. Random noise attenuation based on residual learning of deep convolutional neural network. In: SEG Technical Program Expanded Abstracts 2018. Society of Exploration Geophysicists, pp. 1986-1990.

[189] Simonyan, K., Zisserman, A., 2014. Very deep convolutional networks for large-scale image recognition. arXiv preprint arXiv:1409.1556.

[190] Sivakumar, K., Desai, U. B., 1993. Image restoration using a multilayer perceptron with a multilevel sigmoidal function. IEEE Transactions on Signal Processing 41 (5), 2018-2022.

[191] Soltanayev, S., Chun, S. Y., 2018. Training deep learning based denoisers without ground truth data. In: Advances in Neural Information Processing Systems. pp. 3257-3267.

[192] Song, Y., Zhu, Y., Du, X., 2019. Dynamic residual dense network for image denoising. Sensors 19 (17), 3809.

[193] Stone, J. E., Gohara, D., Shi, G., 2010. Opencl: A parallel programming standard for heterogeneous computing systems. Computing in science \& engineering 12 (3), 66.

[194] Su, Y., Lian, Q., Zhang, X., Shi, B., Fan, X., 2019. Multi-scale cross-path concatenation residual network for poisson denoising. IET Image Processing.

[195] Sun, X., Kottayil, N. K., Mukherjee, S., Cheng, I., 2018. Adversarial training for dual-stage image denoising enhanced with feature matching. In: International Conference on Smart Multimedia. Springer, pp. 357-366.

[196] Szegedy, C., Liu, W., Jia, Y., Sermanet, P., Reed, S., Anguelov, D., Erhan, D., Vanhoucke, V., Rabinovich, A., 2015. Going deeper with convolutions. In: Proceedings of the IEEE Conference on Computer Vision and Pattern Recognition. pp. 1-9.

[197] Tai, Y., Yang, J., Liu, X., Xu, C., 2017. Memnet: A persistent memory network for image restoration. In: Proceedings of the IEEE international Conference on Computer Vision. pp. 4539-4547.

[198] Tamura, S., 1989. An analysis of a noise reduction neural network. In: International Conference on Acoustics, Speech, and Signal Processing,. IEEE, pp. 2001-2004.

[199] Tan, H., Xiao, H., Lai, S., Liu, Y., Zhang, M., 2019. Deep residual learning for burst denoising. In: 2019 IEEE 4th International Conference on Image, Vision and Computing (ICIVC). IEEE, pp. 156-161.

[200] Tao, L., Zhu, C., Song, J., Lu, T., Jia, H., Xie, X., 2017. Low-light image enhancement using cnn and bright channel prior. In: 2017 IEEE International Conference on Image Processing (ICIP). IEEE, pp. 3215-3219.

[201] Tao, L., Zhu, C., Xiang, G., Li, Y., Jia, H., Xie, X., 2017. Llcnn: A convolutional neural network for low-light image enhancement. In: 2017 IEEE Visual Communications and Image Processing (VCIP). IEEE, pp. 1-4.

[202] Tassano, M., Delon, J., Veit, T., 2019. An analysis and implementation of the ffdnet image denoising method. Image Processing On Line 9, 1-25.

[203] Tassano, M., Delon, J., Veit, T., 2019. Dvdnet: A fast network for deep video denoising. In: 2019 IEEE International Conference on Image Processing (ICIP). IEEE, pp. 1805-1809.

[204] Tian, C., Xu, Y., Fei, L., Wang, J., Wen, J., Luo, N., 2019. Enhanced cnn for image denoising. CAAI Transactions on Intelligence Technology 4 (1), 17-23.

[205] Tian, C., Xu, Y., Fei, L., Yan, K., 2018. Deep learning for image denoising: a survey. In: International Conference on Genetic and Evolutionary Computing. Springer, pp. 563-572.

[206] Tian, C., Xu, Y., Li, Z., Zuo, W., Fei, L., Liu, H., 2020. Attention-guided cnn for image denoising. Neural Networks.

[207] Tian, C., Xu, Y., Zuo, W., 2020. Image denoising using deep cnn with batch renormalization. Neural Networks 121, 461-473.

[208] Tian, C., Xu, Y., Zuo, W., Du, B., Lin, C.-W., Zhang, D., 2020. Designing and training of a dual cnn for image 
denoising. arXiv preprint arXiv:2007.03951.

[209] Tian, C., Xu, Y., Zuo, W., Zhang, B., Fei, L., Lin, C.-W., 2020. Coarse-to-fine cnn for image super-resolution. IEEE Transactions on Multimedia.

[210] Tian, C., Zhuge, R., Wu, Z., Xu, Y., Zuo, W., Chen, C., Lin, C.-W., 2020. Lightweight image super-resolution with enhanced cnn. Knowledge-Based Systems, 106235.

[211] Tran, L., Yin, X., Liu, X., 2017. Disentangled representation learning gan for pose-invariant face recognition. In: Proceedings of the IEEE Conference on Computer Vision and Pattern Recognition. pp. 1415-1424.

[212] Tripathi, S., Lipton, Z. C., Nguyen, T. Q., 2018. Correction by projection: Denoising images with generative adversarial networks. arXiv preprint arXiv:1803.04477.

[213] Uchida, K., Tanaka, M., Okutomi, M., 2018. Non-blind image restoration based on convolutional neural network. In: 2018 IEEE 7th Global Conference on Consumer Electronics (GCCE). IEEE, pp. 40-44.

[214] Vedaldi, A., Lenc, K., 2015. Matconvnet: Convolutional neural networks for matlab. In: Proceedings of the 23rd ACM International Conference on Multimedia. ACM, pp. 689-692.

[215] Vogel, C., Pock, T., 2017. A primal dual network for low-level vision problems. In: German Conference on Pattern Recognition. Springer, pp. 189-202.

[216] Wang, C., Zhou, S. K., Cheng, Z., 2020. First image then video: A two-stage network for spatiotemporal video denoising. arXiv preprint arXiv:2001.00346.

[217] Wang, H., Wang, Q., Gao, M., Li, P., Zuo, W., 2018. Multi-scale location-aware kernel representation for object detection. In: Proceedings of the IEEE Conference on Computer Vision and Pattern Recognition. pp. 1248-1257.

[218] Wang, T., Qin, Z., Zhu, M., 2017. An elu network with total variation for image denoising. In: International Conference on Neural Information Processing. Springer, pp. 227-237.

[219] Wang, T., Sun, M., Hu, K., 2017. Dilated deep residual network for image denoising. In: 2017 IEEE 29th International Conference on Tools with Artificial Intelligence (ICTAI). IEEE, pp. 1272-1279.

[220] Wang, X., Dai, F., Ma, Y., Guo, J., Zhao, Q., Zhang, Y., 2019. Near-infrared image guided neural networks for color image denoising. In: ICASSP 2019-2019 IEEE International Conference on Acoustics, Speech and Signal Processing (ICASSP). IEEE, pp. 3807-3811.

[221] Wei, J., Xia, Y., Zhang, Y., 2019. M3net: A multi-model, multi-size, and multi-view deep neural network for brain magnetic resonance image segmentation. Pattern Recognition 91, 366-378.

[222] Wen, J., Xu, Y., Liu, H., 2020. Incomplete multiview spectral clustering with adaptive graph learning. IEEE Transactions on Cybernetics 50 (4), 1418-1429.

[223] Wen, J., Zhang, Z., Zhang, Z., Fei, L., Wang, M., 2020. Generalized incomplete multiview clustering with flexible locality structure diffusion. IEEE Transactions on Cybernetics.

[224] Wu, D., Ren, H., Li, Q., 2020. Self-supervised dynamic ct perfusion image denoising with deep neural networks. arXiv preprint arXiv:2005.09766.

[225] Wu, S., Xu, Y., 2019. Dsn: A new deformable subnetwork for object detection. IEEE Transactions on Circuits and Systems for Video Technology.

[226] Xia, Z., Perazzi, F., Gharbi, M., Sunkavalli, K., Chakrabarti, A., 2019. Basis prediction networks for effective burst denoising with large kernels. arXiv preprint arXiv:1912.04421.

[227] Xiao, P., Guo, Y., Zhuang, P., 2018. Removing stripe noise from infrared cloud images via deep convolutional networks. IEEE Photonics Journal 10 (4), 1-14.

[228] Xiao, X., Xiong, N. N., Lai, J., Wang, C.-D., Sun, Z., Yan, J., 2019. A local consensus index scheme for random-valued impulse noise detection systems. IEEE Transactions on Systems, Man, and Cybernetics: Systems.

[229] Xie, W., Li, Y., Jia, X., 2018. Deep convolutional networks with residual learning for accurate spectral-spatial denoising. Neurocomputing 312, 372-381.

[230] Xu, J., Li, H., Liang, Z., Zhang, D., Zhang, L., 2018. Real-world noisy image denoising: A new benchmark. arXiv preprint arXiv:1804.02603.

[231] Xu, J., Zhang, L., Zhang, D., 2018. External prior guided internal prior learning for real-world noisy image denoising. IEEE Transactions on Image Processing 27 (6), 2996-3010.

[232] Xu, J., Zhang, L., Zhang, D., 2018. A trilateral weighted sparse coding scheme for real-world image denoising. 
In: Proceedings of the European Conference on Computer Vision (ECCV). pp. 20-36.

[233] Xu, J., Zhang, L., Zuo, W., Zhang, D., Feng, X., 2015. Patch group based nonlocal self-similarity prior learning for image denoising. In: Proceedings of the IEEE International Conference on Computer Vision. pp. $244-252$.

[234] Xu, Q., Zhang, C., Zhang, L., 2015. Denoising convolutional neural network. In: 2015 IEEE International Conference on Information and Automation. IEEE, pp. 1184-1187.

[235] Xu, X., Li, M., Sun, W., 2019. Learning deformable kernels for image and video denoising. arXiv preprint arXiv:1904.06903.

[236] Yan, H., Tan, V., Yang, W., Feng, J., 2019. Unsupervised image noise modeling with self-consistent gan. arXiv preprint arXiv:1906.05762.

[237] Yang, D., Sun, J., 2017. Bm3d-net: A convolutional neural network for transform-domain collaborative filtering. IEEE Signal Processing Letters 25 (1), 55-59.

[238] Yang, J., Chu, D., Zhang, L., Xu, Y., Yang, J., 2013. Sparse representation classifier steered discriminative projection with applications to face recognition. IEEE Transactions on Neural Networks and Learning Systems 24 (7), 1023-1035.

[239] Yang, J., Liu, X., Song, X., Li, K., 2017. Estimation of signal-dependent noise level function using multicolumn convolutional neural network. In: 2017 IEEE International Conference on Image Processing (ICIP). IEEE, pp. 2418-2422.

[240] Yang, J., Zhang, L., Xu, Y., Yang, J.-y., 2012. Beyond sparsity: The role of 11-optimizer in pattern classification. Pattern Recognition 45 (3), 1104-1118.

[241] Yao, Y., Wu, X., Zhang, L., Shan, S., Zuo, W., 2018. Joint representation and truncated inference learning for correlation filter based tracking. In: Proceedings of the European Conference on Computer Vision (ECCV). pp. 552-567.

[242] Ye, J. C., Han, Y., Cha, E., 2018. Deep convolutional framelets: A general deep learning framework for inverse problems. SIAM Journal on Imaging Sciences 11 (2), 991-1048.

[243] Yeh, R. A., Lim, T. Y., Chen, C., Schwing, A. G., Hasegawa-Johnson, M., Do, M., 2018. Image restoration with deep generative models. In: 2018 IEEE International Conference on Acoustics, Speech and Signal Processing (ICASSP). IEEE, pp. 6772-6776.

[244] Yi, D., Lei, Z., Liao, S., Li, S. Z., 2014. Learning face representation from scratch. arXiv preprint arXiv:1411.7923.

[245] Yu, A., Liu, X., Wei, X., Fu, T., Liu, D., 2018. Generative adversarial networks with dense connection for optical coherence tomography images denoising. In: 2018 11th International Congress on Image and Signal Processing, BioMedical Engineering and Informatics (CISP-BMEI). IEEE, pp. 1-5.

[246] Yu, S., Ma, J., Wang, W., 2019. Deep learning for denoising. Geophysics 84 (6), V333-V350.

[247] Yuan, D., Fan, N., He, Z., 2020. Learning target-focusing convolutional regression model for visual object tracking. Knowledge-Based Systems, 105526.

[248] Yuan, D., Li, X., He, Z., Liu, Q., Lu, S., 2020. Visual object tracking with adaptive structural convolutional network. Knowledge-Based Systems, 105554.

[249] Yuan, Q., Zhang, Q., Li, J., Shen, H., Zhang, L., 2018. Hyperspectral image denoising employing a spatialspectral deep residual convolutional neural network. IEEE Transactions on Geoscience and Remote Sensing 57 (2), 1205-1218.

[250] Yuan, Y., Liu, S., Zhang, J., Zhang, Y., Dong, C., Lin, L., 2018. Unsupervised image super-resolution using cycle-in-cycle generative adversarial networks. In: Proceedings of the IEEE Conference on Computer Vision and Pattern Recognition Workshops. pp. 701-710.

[251] Yue, Z., Yong, H., Zhao, Q., Meng, D., Zhang, L., 2019. Variational denoising network: Toward blind noise modeling and removal. In: Advances in Neural Information Processing Systems. pp. 1688-1699.

[252] Zamparelli, M., 1997. Genetically trained cellular neural networks. Neural Networks 10 (6), $1143-1151$.

[253] Zarshenas, A., Suzuki, K., 2018. Deep neural network convolution for natural image denoising. In: 2018 IEEE International Conference on Systems, Man, and Cybernetics (SMC). IEEE, pp. 2534-2539.

[254] Zha, Z., Yuan, X., Yue, T., Zhou, J., 2018. From rank estimation to rank approximation: Rank residual constraint for image denoising. arXiv preprint arXiv:1807.02504.

[255] Zhang, B., Jin, S., Xia, Y., Huang, Y., Xiong, Z., 2020. Attention mechanism enhanced kernel prediction 
networks for denoising of burst images. In: ICASSP 2020-2020 IEEE International Conference on Acoustics, Speech and Signal Processing (ICASSP). IEEE, pp. 2083-2087.

[256] Zhang, F., Liu, D., Wang, X., Chen, W., Wang, W., 2018. Random noise attenuation method for seismic data based on deep residual networks. In: International Geophysical Conference, Beijing, China, 24-27 April 2018. Society of Exploration Geophysicists and Chinese Petroleum Society, pp. 1774-1777.

[257] Zhang, J., Ghanem, B., 2018. Ista-net: Interpretable optimization-inspired deep network for image compressive sensing. In: Proceedings of the IEEE Conference on Computer Vision and Pattern Recognition. pp. 1828-1837.

[258] Zhang, K., Zuo, W., Chen, Y., Meng, D., Zhang, L., 2017. Beyond a gaussian denoiser: Residual learning of deep cnn for image denoising. IEEE Transactions on Image Processing 26 (7), 3142-3155.

[259] Zhang, K., Zuo, W., Gu, S., Zhang, L., 2017. Learning deep cnn denoiser prior for image restoration. In: Proceedings of the IEEE Conference on Computer Vision and Pattern Recognition. pp. 3929-3938.

[260] Zhang, K., Zuo, W., Zhang, L., 2018. Ffdnet: Toward a fast and flexible solution for cnn-based image denoising. IEEE Transactions on Image Processing 27 (9), 4608-4622.

[261] Zhang, K., Zuo, W., Zhang, L., 2018. Learning a single convolutional super-resolution network for multiple degradations. In: Proceedings of the IEEE Conference on Computer Vision and Pattern Recognition. pp. 32623271 .

[262] Zhang, K., Zuo, W., Zhang, L., 2019. Deep plug-and-play super-resolution for arbitrary blur kernels. In: Proceedings of the IEEE Conference on Computer Vision and Pattern Recognition. pp. 1671-1681.

[263] Zhang, L., Wu, X., Buades, A., Li, X., 2011. Color demosaicking by local directional interpolation and nonlocal adaptive thresholding. Journal of Electronic imaging 20 (2), 023016.

[264] Zhang, L., Zhang, L., Mou, X., Zhang, D., 2011. Fsim: A feature similarity index for image quality assessment. IEEE transactions on Image Processing 20 (8), 2378-2386.

[265] Zhang, L., Zuo, W., 2017. Image restoration: From sparse and low-rank priors to deep priors [lecture notes]. IEEE Signal Processing Magazine 34 (5), 172-179.

[266] Zhang, M., Zhang, F., Liu, Q., Wang, S., 2019. Vst-net: Variance-stabilizing transformation inspired network for poisson denoising. Journal of Visual Communication and Image Representation 62, 12-22.

[267] Zhang, Z., Geiger, J., Pohjalainen, J., Mousa, A. E.-D., Jin, W., Schuller, B., 2018. Deep learning for environmentally robust speech recognition: An overview of recent developments. ACM Transactions on Intelligent Systems and Technology (TIST) 9 (5), 49.

[268] Zhang, Z., Wang, L., Kai, A., Yamada, T., Li, W., Iwahashi, M., 2015. Deep neural network-based bottleneck feature and denoising autoencoder-based dereverberation for distant-talking speaker identification. EURASIP Journal on Audio, Speech, and Music Processing 2015 (1), 12.

[269] Zhao, D., Ma, L., Li, S., Yu, D., 2019. End-to-end denoising of dark burst images using recurrent fully convolutional networks. arXiv preprint arXiv:1904.07483.

[270] Zhao, H., Shao, W., Bao, B., Li, H., 2019. A simple and robust deep convolutional approach to blind image denoising. In: Proceedings of the IEEE International Conference on Computer Vision Workshops. pp. 0-0.

[271] Zheng, Y., Duan, H., Tang, X., Wang, C., Zhou, J., 2019. Denoising in the dark: Privacy-preserving deep neural network based image denoising. IEEE Transactions on Dependable and Secure Computing.

[272] ZhiPing, Q., YuanQi, Z., Yi, S., XiangBo, L., 2018. A new generative adversarial network for texture preserving image denoising. In: 2018 Eighth International Conference on Image Processing Theory, Tools and Applications (IPTA). IEEE, pp. 1-5.

[273] Zhou, Y., Chellappa, R., Jenkins, B., 1987. A novel approach to image restoration based on a neural network. In: Proceedings of the International Conference on Neural Networks, San Diego, California.

[274] Zoran, D., Weiss, Y., 2011. From learning models of natural image patches to whole image restoration. In: 2011 International Conference on Computer Vision. IEEE, pp. 479-486.

[275] Zuo, W., Zhang, L., Song, C., Zhang, D., Gao, H., 2014. Gradient histogram estimation and preservation for texture enhanced image denoising. IEEE Transactions on Image Processing 23 (6), 2459-2472. 